\title{
Low-level Software Security by Example
}

\author{
Úlfar Erlingsson ${ }^{1}$, Yves Younan ${ }^{2}$, and Frank Piessens ${ }^{2}$ \\ $1_{\text {Microsoft Research, Silicon Valley }}$ \\ ${ }^{1}$ Reykjavík University, Iceland \\ ${ }^{2}$ Katholieke Universiteit Leuven, Belgium
}

\begin{abstract}
Computers are often subject to external attacks that aim to control software behavior. Typically, such attacks arrive as data over a regular communication channel and, once resident in program memory, trigger pre-existing, low-level software vulnerabilities. By exploiting such flaws, these low-level attacks can subvert the execution of the software and gain control over its behavior. The combined effects of these attacks make them one of the most pressing challenges in computer security. As a result, in recent years, many mechanisms have been proposed for defending against these attacks.

This chapter aims to provide insight in low-level software attack and defense techniques by discussing 4 examples of attacks that are representative of the major types of attacks on $\mathrm{C}$ and $\mathrm{C}++$ software, and 4 examples of defenses selected because of their effectiveness, wide applicability and low enforcement overhead. Attacks and defenses are described in enough detail to be understood even by readers without a background in software security, and with-out a natural inclination for crafting malicious attacks.

Throughout, the attacks and defenses are placed in perspective by showing how they are both facilitated by the gap between the semantics of the high-level language of the software under attack, and the low-level semantics of machine code and the hardware on which the software executes.
\end{abstract}

\section{Introduction}

Software vulnerabilities are software bugs that can be triggered by an attacker with possibly disastrous consequences. This introductory section provides more background about such vulnerabilities, why they are so hard to eliminate, and how they can be introduced in a software system. Both attacking such vulnerabilities, and defending against such attacks depends on low-level details of the software and machine under attack, and this section ends with a note on the presentation of such low-level details in this chapter.

\subsection{The difficulty of eliminating low-level vulnerabilities}

Figure 1 is representative of the attacks and defenses presented in this tutorial. The attacks in Sect. 2 all exploit vulnerabilities similar to that in Fig. 1(a), 


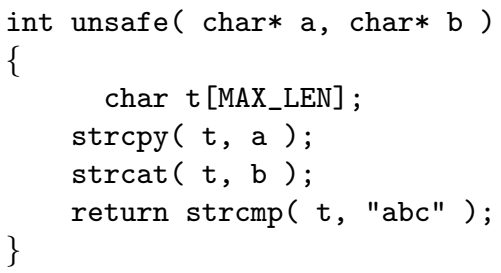

(a) An unchecked C function.

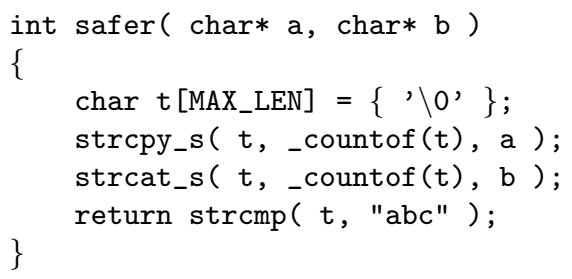

(b) A safer version of the function.

Fig. 1. Two C functions that both compare whether the concatenation of two input strings is the string "abc". The first, unchecked function (a) contains a security vulnerability if the inputs are untrusted. The second function (b) is not vulnerable in this manner, since it uses new $\mathrm{C}$ library functions that perform validity checks against the lengths of buffers Modern compilers will warn about the use of older, less safe library functions, and strongly suggest the use of their newer variants

where a buffer overflow may be possible. For the most part, the defenses in Sect. 3 use techniques like those in Fig. 1(b) and prevent exploits by maintaining additional information, validating that information with runtime checks, and halting execution if such a check fails.

Unfortunately, unlike in Fig. 1, it is often not so straightforward to modify existing source code to use new, safer methods of implementing its functionality. For most code there may not be a direct correspondence between well-known, unsafe library functions and their newer, safer versions. Indeed, existing code can easily be unsafe despite not using any library routines, and vulnerabilities are often obscured by pointer arithmetic or complicated data-structure traversal. (To clarify this point, it is worth comparing the code in Fig. 1 with the code in Fig. 3, on page 5, where explicit loops implement the same functionality.)

Furthermore, manual attempts to remove software vulnerabilities may give a false sense of security, since they do not always succeed and can sometimes introduce new bugs. For example, a programmer that intends to eliminate buffer overflows in the code of Fig. 1(a) might change the strcpy and strcat function calls as in Fig. 1(b), but fail to initialize t to be the empty string at the start of the function. In this case, the strcmp comparison will be against the unmodified array $t$, if both strings $a$ and $b$ are longer than MAX_LEN.

Thus, a slight omission from Fig. 1(b) would leave open the possibility of an exploitable vulnerability as a result of the function reporting that the concatenation of the inputs strings is "abc", even in cases when this is false. In particular, this may occur when, on entry to the function, the array $t$ contains "abc" as a residual data value from a previous invocation of the function.

Low-level software security vulnerabilities continue to persist due to technical reasons, as well as practical engineering concerns such as the difficulties involved in modifying legacy software. The state of the art in eliminating these vulnerabilities makes use of code review, security testing, and other manual software engineering processes, as well as automatic analyses that can discover vulnerabilities [21]. Furthermore, best practice also acknowledges that some vulnerabilities 
are likely to remain, and make those vulnerabilities more difficult to exploit by applying defenses like those in this tutorial.

\subsection{The assumptions underlying software, attacks, and defenses}

Programmers make many assumptions when creating software, both implicitly and explicitly. Some of these assumptions are valid, based on the semantics of the high-level language. For instance, C programmers may assume that execution does not start at an arbitrary place within a function, but at the start of that function.

Programmers may also make questionable assumptions, such as about the execution environment of their software. For instance, software may be written without concurrency in mind, or in a manner that is dependent on the address encoding in pointers, or on the order of heap allocations. Any such assumptions hinder portability, and may result in incorrect execution when the execution environment changes even slightly.

Finally, programmers may make invalid, mistaken assumptions. For example, in $\mathrm{C}$, programmers may assume that the int type behaves like a true, mathematical integer, or that a memory buffer is large enough for the size of the content it may ever need to hold. All of the above types of assumptions are relevant to low-level software security, and each may make the software vulnerable to attack.

At the same time, attackers also make assumptions, and low-level software attacks rely on a great number of specific properties about the hardware and software architecture of their target. Many of these assumptions involve details about names and the meaning of those names, such as the exact memory addresses of variables or functions and how they are used in the software. These assumptions also relate to the software's execution environment, such as the hardware instruction set architecture and its machine-code semantics.

For example, the Internet Worm of 1988 was successful in large part because of an attack that depended on the particulars of the commonly-deployed VAX hardware architecture, the 4 BSD operating system, and the fingerd service. On other systems that were popular at the time, that same attack failed in a manner that only crashed the fingerd service, due to the differences in instruction sets and memory layouts [39]. In this manner, attack code is often fragile to the point where even the smallest change prevents the attacker from gaining control, but crashes the target software - effecting a denial-of-service attack.

Defense mechanisms also have assumptions, including assumptions about the capabilities of the attacker, about the likelihood of different types of attacks, about the properties of the software being defended, and about its execution environment. In the attacks and defenses that follow, a note will be made of the assumptions that apply in each case. Also, many defenses (including most of the ones in this tutorial) assume that denial-of-service is not the attacker's goal, and halt the execution of the target software upon the failure of runtime validity checks. 


\subsection{The presentation of technical details in this chapter}

The presentation in this chapter assumes a basic knowledge of programming languages like $\mathrm{C}$, and their compilation, as might be acquired in an introductory course on compilers. For the most part, relevant technical concepts are introduced when needed.

As well as giving a number of examples of vulnerable $\mathrm{C}$ software, this chapter shows many details relating to software execution, such as machine code and execution stack content. Throughout, the details shown will reflect software execution on one particular hardware architecture - a 32-bit x86, such as the IA-32 [11] — but demonstrate properties that also apply to most other hardware platforms.

The examples show many concrete, hexadecimal values and - in order to avoid confusion - the reader should remember that on the little-endian $\mathrm{x} 86$, when four bytes are displayed as a 32-bit integer value, their printed order will be reversed from the order of the bytes in memory. Thus, if the hexadecimal bytes Oxaa, Oxbb, Oxcc, and 0xdd occur in memory, in that order, then those bytes encode the 32-bit integer 0xddccbbaa.

\section{A selection of low-level attacks on $\mathrm{C}$ software}

This section presents four low-level software attacks in full detail and explains how each attack invalidates a property of target software written in the $\mathrm{C}$ language. The attacks are carefully chosen to be representative of four major classes of attacks: stack-based buffer overflows, heap-based buffer overflows, jump-tolibc attacks, and data-only attacks.

No examples are given below of a "format-string attack" or of an "integeroverflow vulnerability". Format-string vulnerabilities are particularly simple to eliminate [12]; therefore, although they have received a great deal of attention in the past, they are no longer a significant, practical concern in well-engineered

software. Integer-overflow vulnerabilities [8] do still exist, and are increasingly being exploited, but only as a first step towards attacks like those described below. In this section, Attack 4 is one example where an integer overflow might be the first step in the exploit crafted by the attacker.

As further reading, the survey of Pincus and Baker gives a good general overview of low-level software attacks like those described in this section [34].

\subsection{Attack 1: Corruption of a function return address on the stack}

It is natural for $\mathrm{C}$ programmers to assume that, if a function is invoked at a particular call site and runs to completion without throwing an exception, then that function will return to the instruction immediately following that same, particular call site.

Unfortunately, this may not be the case in the presence of software bugs. For example, if the invoked function contains a local array, or buffer, and writes into 


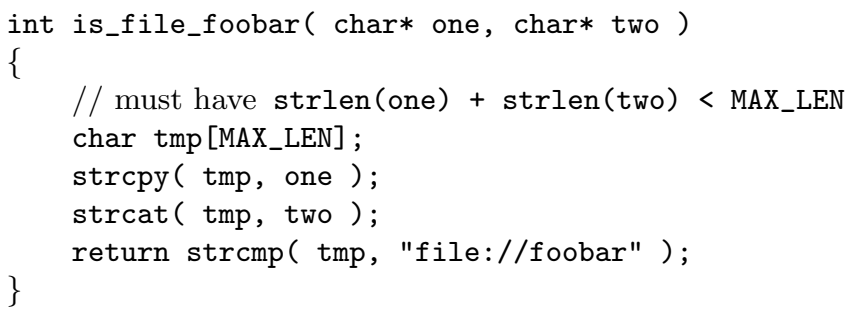

Fig. 2. A $\mathrm{C}$ function that compares the concatenation of two input strings against "file://foobar". This function contains a typical stack-based buffer overflow vulnerability: if the input strings can be chosen by an attacker, then the attacker can direct machine-code execution when the function returns

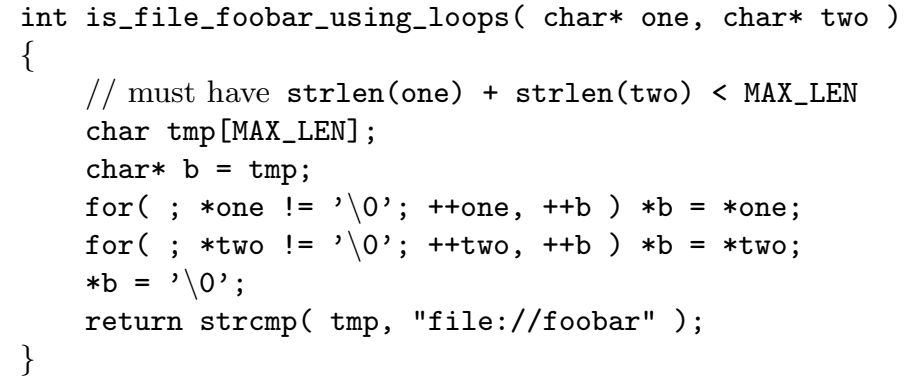

Fig. 3. A version of the $\mathrm{C}$ function in Fig. 2 that copies and concatenates strings using pointer manipulation and explicit loops. This function is also vulnerable to the same stack-based buffer overflow attacks, even though it does not invoke strcpy or strcat or other $\mathrm{C}$ library functions that are known to be difficult to use safely

that buffer are not correctly guarded, then the return address on the stack may be overwritten and corrupted. In particular, this may happen if the software copies to the buffer data whose length is larger than the buffer size, in a buffer overflow.

Furthermore, if an attacker controls the data used by the function, then the attacker may be able to trigger such corruption, and change the function return address to an arbitrary value. In this case, when the function returns, the attacker can direct execution to code of their choice and gain full control over subsequent behavior of the software. Figures 2 and 3 show examples of $\mathrm{C}$ functions that are vulnerable to this attack.

This attack, sometimes referred to as return-address clobbering, is probably the best known exploit of a low-level software security vulnerability; it dates back to before 1988, when it was used in the fingerd exploit of the Internet Worm. Indeed, until about a decade ago, this attack was seen by many as the only significant low-level attack on software compiled from $\mathrm{C}$ and $\mathrm{C}++$, and "stackbased buffer overflow" were widely considered a synonym for such attacks. More recently, this attack has not been as prominent, in part because other methods 


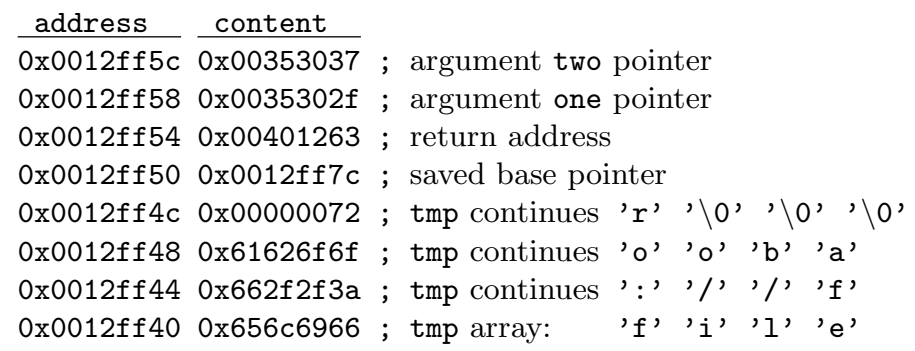

Fig. 4. A snapshot of an execution stack for the functions in Figs. 2 and 3, where the size of the tmp array is 16 bytes. This snapshot shows the stack just before executing the return statement. Argument one is "file://", and argument two is "foobar", and the concatenation of those strings fits in the tmp array. (Stacks are traditionally displayed with the lowest address at the bottom, as is done here and throughout this chapter)

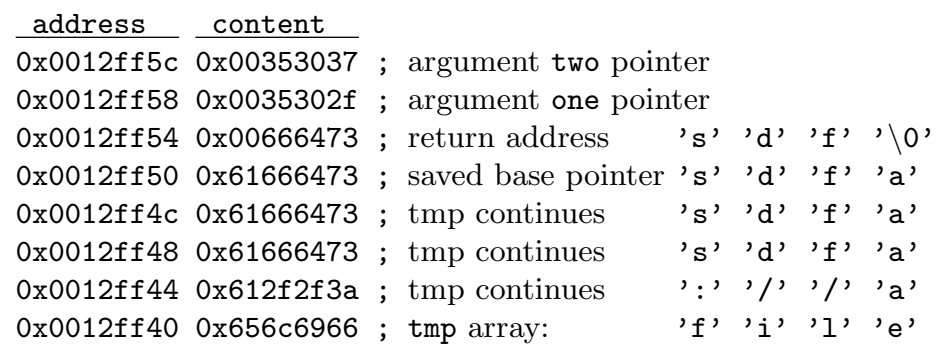

Fig. 5. An execution-stack snapshot like that in Fig. 4, but where argument one is "file://" and argument two is "asdfasdfasdfasdf". The concatenation of the argument strings has overflowed the tmp array and the function return address is now determined by the last few characters of the two string

of attack have been widely publicized, but also in part because the underlying vulnerabilities that enable return-address clobbering are slowly being eliminated (e.g., through the adoption of newer, safer C library functions).

To give a concrete example of this attack, Fig. 4 shows a normal execution stack for the functions in Figs. 2 and 3, and Fig. 5 shows an execution stack for the same code just after an overflow of the local array-potentially caused by an attacker that can choose the contents of the two string provided as input.

Of course, an attacker would choose their input such that the buffer overflow would not caused by "asdfasdfasdfasdf", but another string of bytes. In particular, the attacker might choose $0 \times 48,0 \times f f$, and $0 \times 12$, in order, as the final three character bytes of the two argument string - and thereby arrange for the function return address to have the value $0 \times 0012 \mathrm{f} f 48$. In this case, as soon as the function returns, the hardware instruction pointer would be placed at the second character of the two argument string, and the hardware would start executing the data found there (and chosen by the attacker) as machine code. 


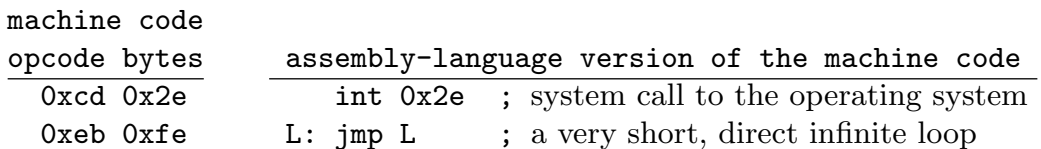

Fig. 6. The simple attack payload used in this chapter; in most examples, the attacker's goal will be to execute this machine code. Of these four bytes, the first two are a x86 int instruction which performs a system call on some platforms, and the second two are an x86 jmp instruction that directly calls itself in an infinite loop (Note that, in the examples, these bytes will sometimes be printed as the integer 0xfeeb2ecd, with the apparent reversal a result of $\mathrm{x} 86$ little-endianness)

In the example under discussion, an attacker would choose their input data so that the machine code for an attack payload would be present at address 0x0012ff48. When the vulnerable function returns, and execution of the attack payload begins, the attacker has gained control of the behavior of the target software. (The attack payload is often called shellcode, since a common goal of an attacker is to launch a "shell" command interpreter under their control.)

In Fig. 5 , the bytes at $0 \times 0012 \mathrm{ff} 48$ are those of the second to fifth characters in the string "asdfasdfasdfasdf", namely 's', 'd', ' $f$ ', and 'a'. When executed as machine code, those bytes do not implement an attack. Instead, as described in Fig. 6, an attacker might choose $0 x c d, 0 x 2 e, 0 x e b$, and $0 x f e$ as a very simple attack payload. Thus, an attacker might call the operating system to enable a dangerous feature, or disable security checks, and avoid detection by keeping the target software running (albeit in a loop).

Return-address clobbering as described above has been a highly successful attack technique - for example, in 2003 it was used to implement the Blaster worm, which affected a majority of Internet users [5]. In the case of Blaster, the vulnerable code was written using explicit loops, much as in Fig. 3. (This was one reason why the vulnerability had not been detected and corrected through automatic software analysis tools, or by manual code reviews.)

\section{Attack 1: Constraints and variants}

Low-level attacks are typically subject to a number of such constraints, and must be carefully written to be compatible with the vulnerability being exploited.

For example, the attack demonstrated above relies on the hardware being willing to execute the data found on the stack as machine code. However, on some systems the stack is not executable, e.g., because those systems implement the defenses described later in this chapter. On such systems, an attacker would have to pursue a more indirect attack strategy, such as those described later, in Attacks 3 and 4.

Another important constraint applies to the above buffer-overflow attacks: the attacker-chosen data cannot contain null bytes, or zeros - since such bytes terminate the buffer overflow and prevent further copying onto the stack. This is a common constraint when crafting exploits of buffer overflows, and applies to most of the attacks in this chapter. It is so common that special tools exist for 


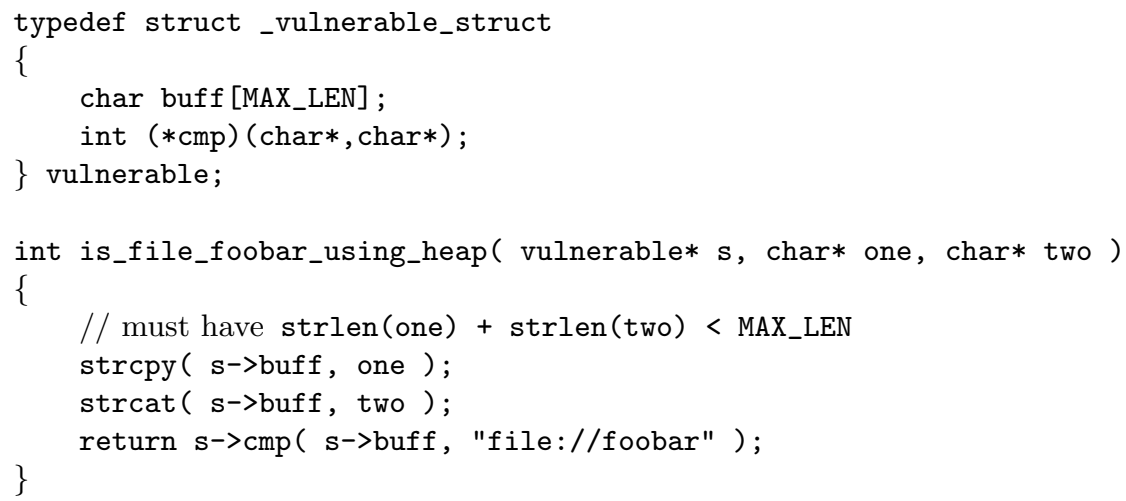

Fig. 7. A C function that sets a heap data structure as the concatenation of two input strings, and compares the result against "file://foobar" using the comparison function for that data structure. This function is vulnerable to a heap-based buffer overflow attack if an attacker can choose either or both of the input strings

creating machine code for attack payloads that do not contain any embedded null bytes, newline characters, or other byte sequences that might terminate the buffer overflow (one such tool is Metasploit [17]).

There are a number of attack methods similar to return-address clobbering, in that they exploit stack-based buffer overflow vulnerabilities to target the function-invocation control data on the stack. Most of these variants add a level of indirection to the techniques described above.

One notable attack variant corrupts the base pointer saved on the stack (see Figs. 4 and 5) and not the return address sitting above it. In this variant, the vulnerable function may return as expected to its caller function, but, when that caller itself returns, it uses a return address that has been chosen by the attacker [27]. Another notable variant of this attack targets $\mathrm{C}$ and $\mathrm{C}++$ exception-handler pointers that reside on the stack, and ensures that the buffer overflow causes an exception - at which point a function pointer of the attacker's choice may be executed [29].

\subsection{Attack 2: Corruption of function pointers stored in the heap}

Software written in $\mathrm{C}$ and $\mathrm{C}++$ often combines data buffers and pointers into the same data structures, or objects, with programmers making a natural assumption that the data values do not affect the pointer values. Unfortunately, this may not be the case in the presence of software bugs. In particular, the pointers may be corrupted as a result of an overflow of the data buffer-regardless whether the data structures or objects reside on stack, or in heap memory. Fig. 7 shows $\mathrm{C}$ code with a function that is vulnerable to such an attack.

To give a concrete example of this attack, Fig. 8 shows the contents of the vulnerable data structure after the function in Fig. 7 has copied data into the buff array using the strcpy and strcmp library functions. Figure 8 shows 


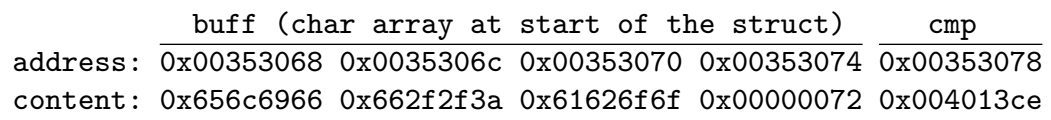

(a) A structure holding "file://foobar" and a pointer to the strcmp function.

address: $\frac{\text { buff (char array at start of the struct) }}{0 \times 00353068 \text { 0x0035306c 0x00353070 0x00353074 }} \frac{\mathrm{cmp}}{0 \times 00353078}$

content: 0x656c6966 0x612f2f3a 0x61666473 0x61666473 0x00666473

(b) After a buffer overflow caused by the inputs "file://" and "asdfasdfasdf".

$\begin{array}{lllll} & \text { buff (char array at start of the struct) } & \text { cmp } \\ \text { address: } & \text { 0x00353068 } 0 x 0035306 \mathrm{c} & \text { 0x00353070 } & \text { ox00353074 } & \text { 0x00353078 } \\ \text { content: } & \text { 0xfeeb2ecd } \text { 0x11111111 } & \text { 0x11111111 } & \text { 0x11111111 } & \text { 0x00353068 }\end{array}$

(c) After a malicious buffer overflow caused by attacker-chosen inputs.

Fig. 8. Three instances of the vulnerable data structure pointed to by $\mathrm{s}$ in Fig. 7, where the size of the buff array is 16 bytes. Both the address of the structure and its 20 bytes of content are shown. In the first instance (a), the buffer holds "file://foobar" and $\mathrm{cmp}$ points to the strcmp function. In the second instance (b), the pointer has been corrupted by a buffer overflow. In the third instance (c), an attacker has selected the input strings so that the buffer overflow has changed the structure data so that the simple attack payload of Fig. 6, page 7 , will be executed

three instances of the data structure contents: as might occur during normal processing, as might occur in an unintended buffer overflow, and, finally, as might occur during an attack. These instances can occur both when the data structure is allocated on the stack, and also when it is allocated on the heap.

In the last instance of Fig. 8, the attacker has chosen the two input strings such that the cmp function pointer has become the address of the start of the data structure. At that address, the attacker has arranged for an attack payload to be present. Thus, when the function in Fig. 7 executes the return statement, and invokes $\mathbf{s}^{->c m p}$, it transfers control to the start of the data structure, which contains data of the attacker's choice. In this case, the attack payload is the four bytes of machine code $0 x c d, 0 x 2 e$, $0 x e b$, and $0 x f e$ described in Fig. 6, page 7, and used throughout this chapter.

It is especially commonplace for $\mathrm{C}++$ code to store object instances on the heap and to combine - within a single object instance - both data buffers that may be overflowed and potentially exploitable pointers. In particular, $\mathrm{C}++$ object instances are likely to contain vtable pointers: a form of indirect function pointers that allow dynamic dispatch of virtual member functions. As a result, $\mathrm{C}++$ software may be particularly vulnerable to heap-based attacks [35].

\section{Attack 2: Constraints and variants}

Heap-based attacks are often constrained by their ability to determine the address of the heap memory that is being corrupted, as can be seen in the exam- 
ples above. This constraint applies in particular, to all indirect attacks, where a heap-based pointer-to-a-pointer is modified. Furthermore, the exact bytes of those addresses may constrain the attacker, e.g., if the exploited vulnerability is that of a string-based buffer overflow, in which case the address data cannot contain null bytes.

The examples above demonstrate attacks where heap-based buffer overflow vulnerabilities are exploited to corrupt pointers that reside within the same data structure or object as the data buffer that is overflowed. There are two important attack variants, not described above, where heap-based buffer overflows are used to corrupt pointers that reside in other structures or objects, or in the heap metadata.

In the first variant, two data structures or objects reside consecutively in heap memory, the initial one containing a buffer that can be overflowed, and the subsequent one containing a direct, or indirect, function pointer. Heap objects are often adjacent in memory like this when they are functionally related and are allocated in order, one immediately after the other. Whenever these conditions hold, attacks similar to the above examples may be possible, by overflowing the buffer in the first object and overwriting the pointer in the second object.

In the second variant, the attack is based on corrupting the metadata of the heap itself through a heap-based buffer overflow, and exploiting that corruption to write an arbitrary value to an arbitrary location in memory. This is possible because heap implementations contain doubly-linked lists in their metadata. An attacker that can corrupt the metadata can thereby choose what is written where. The attacker can then use this capability to write a pointer to the attack payload in the place of any soon-to-be-used function pointer sitting at a known address.

\subsection{Attack 3: Execution of existing code via corrupt pointers}

If software does not contain any code for certain functionality - such as performing floating-point calculations, or making system calls to interact with the network - then the programmers may naturally assume that execution of the software will not result in this behavior, or functionality.

Unfortunately, for $\mathrm{C}$ or $\mathrm{C}++$ software, this assumption may not hold in the face of bugs and malicious attacks, as demonstrated by attacks like those in this chapter. As in the previous two examples of attacks, the attacker may be able to cause arbitrary behavior by direct code injection: by directly modifying the hardware instruction pointer to execute machine code embedded in attackerprovided input data, instead of the original software. However, there are other means for an attacker to cause software to exhibit arbitrary behavior, and these alternatives can be the preferred mode of attack.

In particular, an attacker may find it preferable to craft attacks that execute the existing machine code of the target software in a manner not intended by its programmers. For example, the attacker may corrupt a function pointer to cause the execution of a library function that is unreachable in the original $\mathrm{C}$ or $\mathrm{C}++$ source code written by the programmers - and should therefore, in the compiled 


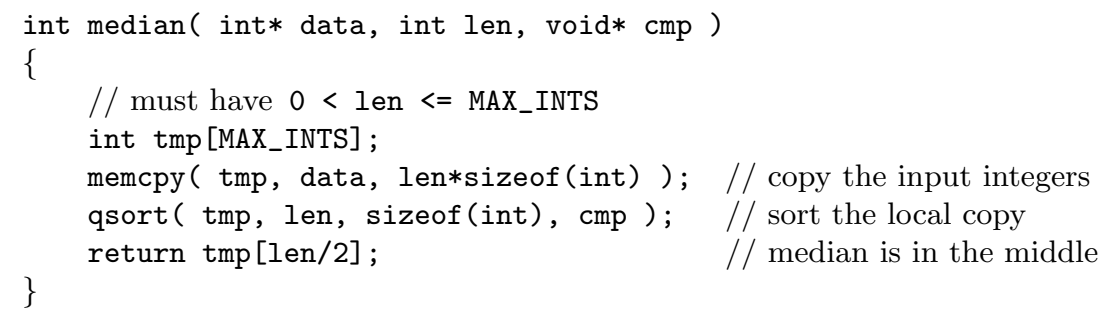

Fig. 9. A C function that computes the median of an array of input integers by sorting a local copy of those integers. This function is vulnerable to a stack-based buffer overflow attack, if an attacker can choose the set of input integers

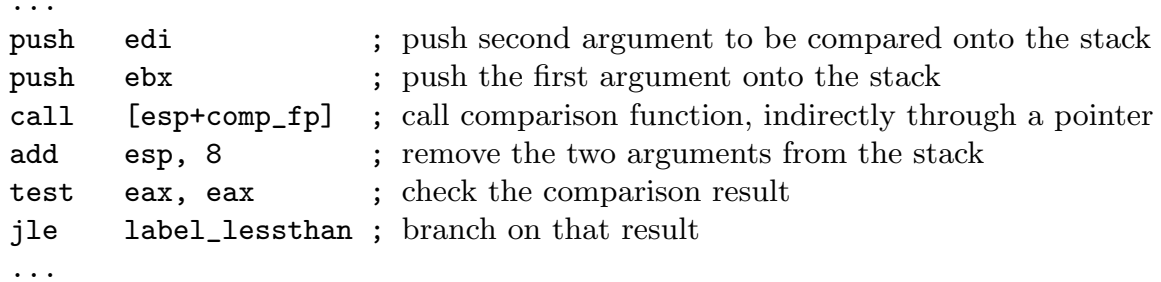

Fig. 10. Machine code fragment from the qsort library function, showing how the comparison operation is called through a function pointer. When qsort is invoked in the median function of Fig. 9, a stack-based buffer overflow attack can make this function pointer hold an arbitrary address

software, be never-executed, dead code. Alternatively, the attacker may arrange for reachable, valid machine code to be executed, but in an unexpected order, or with unexpected data arguments.

This class of attacks is typically referred to as jump-to-libc or return-to$l i b c$ (depending on whether a function pointer or return address is corrupted by the attacker), because the attack often involves directing execution towards machine code in the libc standard C library.

Jump-to-libc attacks are especially attractive when the target software system is based on an architecture where input data cannot be directly executed as machine code. Such architectures are becoming commonplace with the adoption of the defenses such as those described later in this chapter. As a result, an increasingly important class of attacks is indirect code injection: the selective execution of the target software's existing machine code in a manner that enables attacker-chosen input data to be subsequently executed as machine code. Figure 9 shows a $\mathrm{C}$ function that is vulnerable to such an attack.

The function in Fig. 9 actually contains a stack-based buffer overflow vulnerability that can be exploited for various attacks, if an attacker is able to choose the number of input integers, and their contents. In particular, attackers can perform return-address clobbering, as described in Attack 1. However, for this particular function, an attacker can also corrupt the comparison-function pointer 


\begin{tabular}{|c|c|c|}
\hline address & $\begin{array}{l}\text { machine code } \\
\text { opcode bytes }\end{array}$ & assembly-language version of the machine code \\
\hline $0 \times 7 c 971649$ & $0 x 8 b \quad 0 x e 3$ & mov esp, ebx ; change the stack location to ebx \\
\hline $0 \times 7 c 97164 b$ & $0 \times 5 b$ & ; pop ebx from the new stack \\
\hline $0 \times 7 c 97164 c$ & $0 x c 3$ & ; return based on the new stack \\
\hline
\end{tabular}

Fig. 11. Four bytes found within executable memory, in a system library. These bytes encode three machine-code instructions that are useful in the crafting of jump-tolibc attacks. In particular, in an attack on the median function in Fig. 9, these three instructions may be called by the qsort code in Fig. 10, which will change the stack pointer to the start of the local tmp buffer that has been overflowed by the attacker

\begin{tabular}{|c|c|c|c|c|}
\hline $\begin{array}{c}\text { stack } \\
\text { address }\end{array}$ & $\begin{array}{l}\text { normal } \\
\text { stack } \\
\text { contents }\end{array}$ & $\begin{array}{l}\text { benign } \\
\text { overflow } \\
\text { contents } \\
\end{array}$ & $\begin{array}{l}\text { malicious } \\
\text { overflow } \\
\text { contents }\end{array}$ & \\
\hline $0 \times 0012 f f 38$ & $\overline{0 x 004013 e 0}$ & $\overline{0 x 1111110 d}$ & $\overline{0 \times 7 c 971649}$ & ; cmp argument \\
\hline $0 x 0012 f f 34$ & $0 \times 00000001$ & $0 \times 1111110 c$ & $0 \times 1111110 c$ & ; len argument \\
\hline $0 x 0012 f f 30$ & $0 \times 00353050$ & $0 \mathrm{x} 1111110 \mathrm{~b}$ & $0 \mathrm{x} 1111110 \mathrm{~b}$ & ; data argument \\
\hline $0 x 0012 f f 2 c$ & $0 \times 00401528$ & $0 \times 1111110 a$ & Oxfeeb2ecd & ; return address \\
\hline $0 x 0012 f f 28$ & $0 x 0012 f f 4 c$ & $0 \times 11111109$ & $0 \times 70000000$ & ; saved base pointer \\
\hline $0 x 0012 f f 24$ & $0 \times 00000000$ & $0 \times 11111108$ & $0 \times 70000000$ & ; tmp final 4 bytes \\
\hline $0 x 0012 f f 20$ & $0 \times 00000000$ & $0 \times 11111107$ & $0 \times 00000040$ & ; tmp continues \\
\hline $0 x 0012 f f 1 c$ & $0 \times 00000000$ & $0 \times 11111106$ & $0 \times 00003000$ & ; tmp continues \\
\hline $0 x 0012 f f 18$ & $0 \times 00000000$ & $0 \times 11111105$ & $0 \times 00001000$ & ; tmp continues \\
\hline $0 x 0012 f f 14$ & $0 \times 00000000$ & $0 \times 11111104$ & $0 \times 70000000$ & ; tmp continues \\
\hline $0 x 0012 f f 10$ & $0 \times 00000000$ & $0 \times 11111103$ & $0 x 7 c 80978 e$ & ; tmp continues \\
\hline $0 x 0012 f f 0 c$ & $0 \times 00000000$ & $0 \times 11111102$ & $0 \times 7 c 809 a 51$ & ; tmp continues \\
\hline $0 x 0012 f f 08$ & $0 \times 00000000$ & $0 \times 11111101$ & $0 \times 11111101$ & ; tmp buffer starts \\
\hline $0 \times 0012 f f 04$ & $0 \times 00000004$ & $0 \times 00000040$ & $0 \times 00000040$ & ; memcpy length argument \\
\hline $0 \times 0012 f f 00$ & $0 \times 00353050$ & $0 \times 00353050$ & $0 \times 00353050$ & ; memcpy source argument \\
\hline $0 x 0012 f e f c$ & $0 x 0012 f f 08$ & $0 x 0012 f f 08$ & $0 x 0012 f f 08$ & ; memcpy destination arg. \\
\hline
\end{tabular}

Fig. 12. The address and contents of the stack of the median function of Fig. 9, where tmp is eight integers in size. Three versions of the stack contents are shown, as it would appear just after the call to memcpy: a first for input data of the single integer zero, a second for a benign buffer overflow of consecutive integers starting at $0 \times 11111101$, and a third for a malicious jump-to-libc attack that corrupts the comparison function pointer to make qsort call address 0x7c971649 and the machine code in Fig. 11

cmp before it is passed to qsort. In this case, the attacker can gain control of machine-code execution at the point where qsort calls its copy of the corrupted cmp argument. Figure 10 shows the machine code in the qsort library function where this, potentially-corrupted function pointer is called.

To give a concrete example of a jump-to-libc attack, consider the case when the function in Fig. 9 is executed on some versions of the Microsoft Windows operating system. On these systems, the qsort function is implemented as shown in Fig. 10 and the memory address 0x7c971649 holds the four bytes of executable machine code, as shown in Fig. 11. 
// call a function to allocate writable, executable memory at 0x70000000

VirtualAlloc(0x70000000, 0x1000, 0x3000, 0x40);// function at 0x7c809a51

// call a function to write the four-byte attack payload to 0x70000000

InterlockedExchange (0x70000000, 0xfeeb2ecd); // function at 0x7c80978e

// invoke the four bytes of attack payload machine code

$((\operatorname{void}(*)()) 0 \times 70000000)() ; \quad / /$ payload at $0 \times 70000000$

Fig. 13. The jump-to-libc attack activity caused by the maliciously-corrupted stack in Fig. 12, expressed as $\mathrm{C}$ source code. As the corrupted stack is unwound, instead of returning to call sites, the effect is a sequence of function calls, first to functions in the standard Windows library kernel32.dll, and then to the attack payload

On such a system, the buffer overflow may leave the stack looking like that shown in the "malicious overflow contents" column of Fig. 12. Then, when the qsort function is called, it is passed a copy of the corrupted cmp functionpointer argument, which points to a trampoline found within existing, executable machine code. This trampoline is the code found at address 0x7c971649, which is shown in Fig. 11. The effect of calling the trampoline is to, first, set the stack pointer esp to the start address of the tmp array, (which is held in register ebx), second, read a new value for ebx from the first integer in the tmp array, and, third, perform a return that changes the hardware instruction pointer to the address held in the second integer in the tmp array.

The attack subsequently proceeds as follows. The stack is "unwound" one stack frame at a time, as functions return to return addresses. The stack holds data, including return addresses, that has been chosen by the attacker to encode function calls and arguments. As each stack frame is unwound, the return instruction transfers control to the start of a particular, existing library function, and provides that function with arguments.

Figure 13 shows, as $\mathrm{C}$ source code, the sequence of function calls that occur when the stack is unwound. The figure shows both the name and address of the Windows library functions that are invoked, as well as their arguments. The effect of these invocations is to create a new, writable page of executable memory, to write machine code of the attacker's choice to that page, and to transfer control to that attack payload.

After the trampoline code executes, the hardware instruction pointer address is $0 \times 7$ c809a51, which is the start of the Windows library function VirtualAlloc, and the address in the stack pointer is $0 x 0012 f f 10$, the third integer in the tmp array in Fig. 12. As a result, when VirtualAlloc returns, execution will continue at address $0 \times 7 c 80978 \mathrm{e}$, which is the start of the Windows library function InterlockedExchange. Finally, the InterlockedExchange function returns to the address 0x70000000, which at that time holds the attack payload machine code in executable memory.

(This attack is facilitated by two Windows particulars: all Windows processes load the library kernel32.dll into their address space, and the Windows calling 
convention makes library functions responsible for popping their own arguments off the stack. On other systems, the attacker would need to slightly modify the details of the attack.)

\section{Attack 3: Constraints and variants}

A major constraint on jump-to-libc attacks is that the attackers must craft each such attack with a knowledge of the addresses of the target-software machine code that is useful to the attack. An attacker may have difficulty in reliably determining these addresses, for instance because of variability in the versions of the target software and its libraries, or because of variability in the target software's execution environment. Artificially increasing this variability is a useful defense against many types of such attacks, as discussed later in this chapter.

Traditionally, jump-to-libc attacks have targeted the system function in the standard system libraries, which allows the execution of an arbitrary command with arguments, as if typed into a shell command interpreter. This strategy can also be taken in the above attack example, with a few simple changes. However, an attacker may prefer indirect code injection, because it requires launching no new processes or accessing any executable files, both of which may be detected or prevented by system defenses.

For software that may become the target of jump-to-libc attacks, one might consider eliminating any fragment of machine code that may be useful to the attacker, such as the trampoline code shown in Fig. 11. This can be difficult for many practical reasons. For instance, it is difficult to selectively eliminate fragments of library code while, at the same time, sharing the code memory of dynamic libraries between their instances in different processes; however, eliminating such sharing would multiply the resource requirements of dynamic libraries. Also, it is not easy to remove data constants embedded within executable code, which may form instructions useful to an attacker. (Examples of such data constants include the jump tables of $\mathrm{C}$ and $\mathrm{C}++$ switch statements.)

Those difficulties are compounded on hardware architectures that use variablelength sequences of opcode bytes for encoding machine-code instructions. For example, on some versions of Windows, the machine code for a system call is encoded using a two-byte opcode sequence, $0 \mathrm{xcd}, 0 \mathrm{x} 2 \mathrm{e}$, while the five-byte sequence $0 \times 25,0 x c d, 0 \times 2 e, 0 \times 00$, and $0 \times 00$ corresponds to an arithmetic operation (the operation and eax, $0 \times 2 e c d$, in $x 86$ assembly code). Therefore, if an instruction for this particular and operation is present in the target software, then jumping to its second byte can be one way of performing a system call. Similarly, any x86 instruction, including those that read or write memory, may be executed through a jump into the middle of the opcode-byte sequence for some other x86 machine-code instruction.

Indeed, for x86 Linux software, it has been recently demonstrated that it is practical for elaborate jump-to-libc attacks to perform arbitrary functionality while executing only machine-code found embedded within other instructions [36]. Much as in the above example, these elaborate attacks proceed through the unwinding of the stack, but they may also "rewind" the stack in order to 


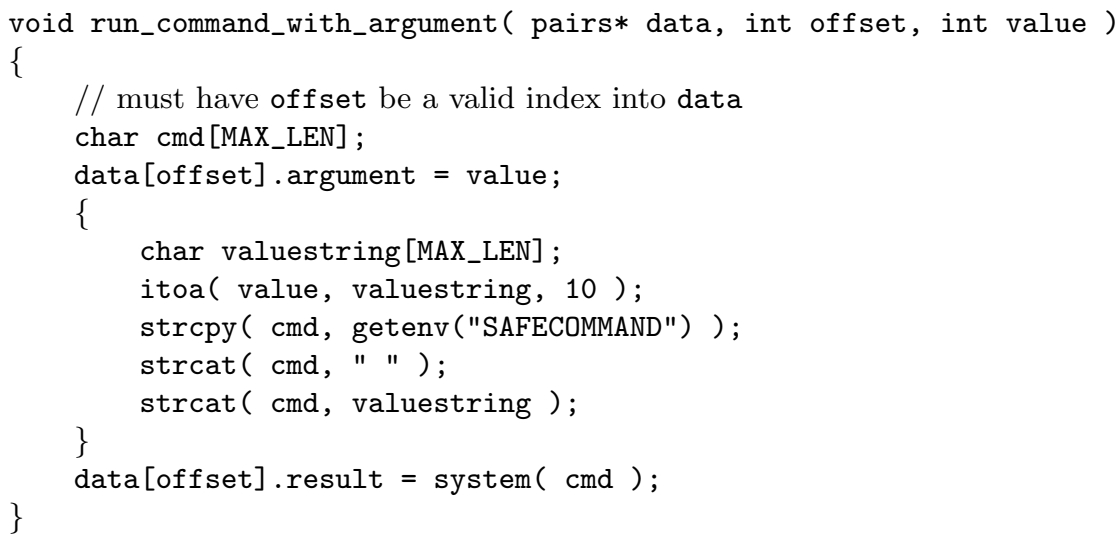

Fig. 14. A $\mathrm{C}$ function that launches an external command with an argument value, and stores in a data structure that value and the result of the command. If the offset and value can be chosen by an attacker, then this function is vulnerable to a data-only attack that allows the attacker to launch an arbitrary external command

encode loops of activity. However, unlike in the above example, these elaborate attacks may allow the attacker to achieve their goals without adding any new, executable memory or machine code the to target software under attack.

Attacks like these are of great practical concern. For example, the flaw in the median function of Fig. 9 is in many ways similar to the recently discovered "animated cursor vulnerability" in Windows [20]. Despite existing, deployed defenses, that vulnerability is subject to a jump-to-libc attack similar to that in the above example.

\subsection{Attack 4: Corruption of data values that determine behavior}

Software programmers make many natural assumptions about the integrity of data. As one example, an initialized global variable may be assumed to hold the same, initial value throughout the software's execution, if it is never written by the software. Unfortunately, for $\mathrm{C}$ or $\mathrm{C}++$ software, such assumptions may not hold in the presence of software bugs, and this may open the door to malicious attacks that corrupt the data that determine the software's behavior.

Unlike the previous attacks in this chapter, data corruption may allow the attacker to achieve their goals without diverting the target software from its expected path of machine-code execution - either directly or indirectly. Such attacks are referred to as data-only, or non-control-data, attacks [10]. In some cases, a single instance of data corruption can be sufficient for an attacker to achieve their goals. Figure 14 shows an example of a $\mathrm{C}$ function that is vulnerable to such an attack.

As a concrete example of a data-only attack, consider how the function in Fig. 14 makes use of the environment string table by calling the getenv routine in the standard $\mathrm{C}$ library. This routine returns the string that is passed to 


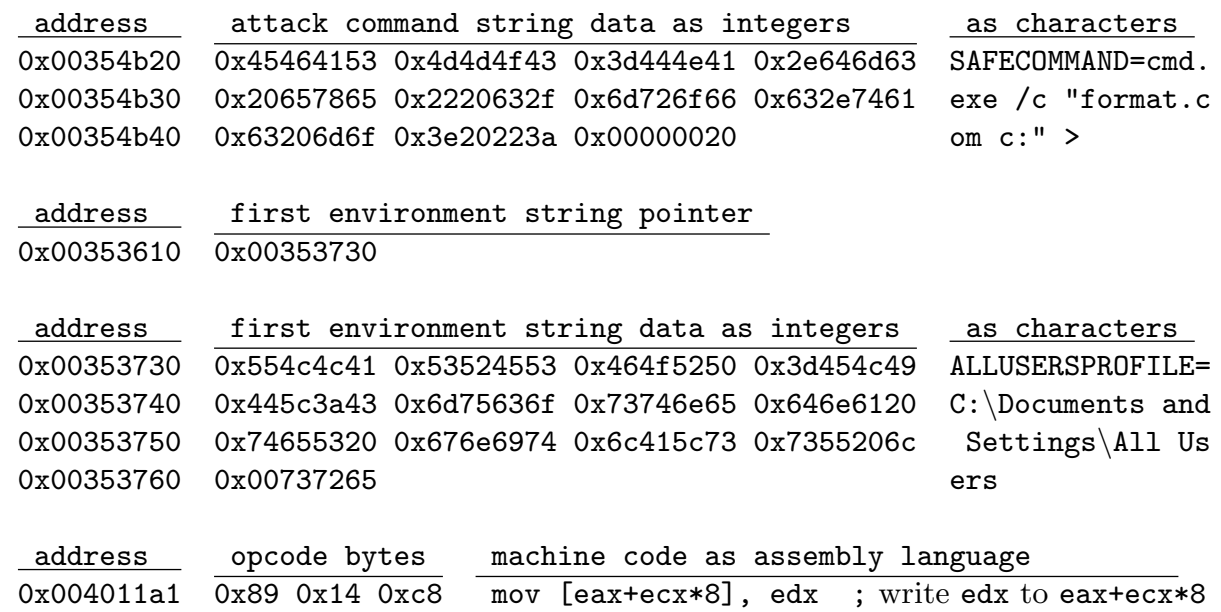

Fig. 15. Some of the memory contents for an execution of the function in Fig. 14, including the machine code for the data [offset] . argument = value; assignment. If the data pointer is $0 \times 004033 \mathrm{e} 0$, the attacker can choose the inputs offset $=0 \times 1 \mathrm{ffea} 046$ and value $=0 \times 00354 \mathrm{~b} 20$, and thereby make the assignment instruction change the first environment string pointer to the "format" command string at the top

another standard routine, system, and this string argument determines what external command is launched. An attacker that is able to control the function's two integer inputs is able to write an arbitrary data value to a nearly-arbitrary location in memory. In particular, this attacker is able to corrupt the table of the environment strings to launch an external command of their choice.

Figure 15 gives the details of such an attack on the function in Fig. 14, by selectively showing the address and contents of data and code memory. In this case, before the attack, the environment string table is an array of pointers starting at address 0x00353610. The first pointer in that table is shown in Fig. 15, as are its contents: a string that gives a path to the "all users profile". In a correct execution of the function, some other pointer in the environment string table would be to a string, such as SAFECOMMAND=safecmd.exe, that determines a safe, external command to be launched by the system library routine.

However, before reading the command string to launch, the machine-code assignment instruction shown in Fig. 15 is executed. By choosing the off set and value inputs to the function, the attacker can make ecx and edx hold arbitrary values. Therefore, the attacker can make the assignment write any value to nearly any address in memory, given knowledge of the data pointer. If the data pointer is $0 \times 004033 \mathrm{e} 0$, then that address plus $8 * 0 \times 1 \mathrm{ffea} 046$ is $0 \times 00353610$, the address of the first environment string pointer. Thus, the attacker is able to write the address of their chosen attack command string, 0x00354b20, at that location. Then, when getenv is called, it will look no further than the first pointer in the environment string table, and return a command string that, when launched, may delete data on the "C:" drive of the target system. 
Several things are noteworthy about this data-only attack and the function in Fig. 14. First, note that there are multiple vulnerabilities that may allow the attacker to choose the off set integer input, ranging from stack-based and heapbased buffer overflows, through integer overflow errors, to a simple programmer mistake that omitted any bounds check. Second, note that although 0x1ffea046 is a positive integer, it effectively becomes negative when multiplied by eight, and the assignment instruction writes to an address before the start of the data array. Finally, note that this attack succeeds even when the table of environment strings is initialized before the execution starts, and the table is never modified by the target software - and when the table should therefore logically be readonly given the semantics of the target software.

\section{Attack 4: Constraints and variants}

There are two major constraints on data-only attacks. First, the vulnerabilities in the target software are likely to allow only certain data, or a certain amount of data to be corrupted, and potentially only in certain ways. For instance, as in the above example, a vulnerability might allow the attacker to change a single, arbitrary four-byte integer in memory to a value of their choice. (Such vulnerabilities exist in some heap implementations, as described on page 10; there, an arbitrary write is possible through the corruption of heap metadata, most likely caused by the overflow of a buffer stored in the heap. Many real-world attacks have exploited this vulnerability, including the GDI+ JPEG attack in Windows $[10,16]$.)

Second, even when an attacker can replace any amount of data with arbitrary values, and that data may be located anywhere, a data-only attack will be constrained by the behavior of the target software when given arbitrary input. For example, if the target software is an arithmetic calculator, a data-only attack might only be able to cause an incorrect result to be computed. However, if the target software embeds any form of an interpreter that performs potentially dangerous operations, then a data-only attack could control the input to that interpreter - allowing the attacker to perform the dangerous operations. The system standard library routine is an example of such an interpreter; many applications, such as Web browsers and document viewers, embed other interpreters for scripting languages.

To date, data-only attacks have not been prominent. Rather, data corruption has been most frequently utilized as one step in other types of attacks, such as direct code injection, or an jump-to-libc attack. This may change with the increased deployment of defenses, including the defenses described below.

\section{Defenses That Preserve High-level Language Properties}

This section presents, in detail, four effective, practical defenses against lowlevel software attacks on x86 machine-code software, and explains how each defense is based on preserving a property of target software written in the $\mathrm{C}$ or 
$\mathrm{C}++$ languages. These defenses are stack canaries, non-executable data, controlflow integrity, and address-space layout randomization. They have been selected based on their efficiency, and ease-of-adoption, as well as their effectiveness.

In particular, this section describes neither defenses based on instruction-set randomization [25], nor defenses based on dynamic information flow tracking, or tainting, or other forms of data-flow integrity enforcement $[9,32]$. Such techniques can offer strong defenses against all the attacks in Sect. 2, although, like the defenses below, they also have limitations and counterattacks. However, these defenses have drawbacks that make their deployment difficult in practice.

For example, unless they are supported by specialized hardware, they incur significant overheads. On unmodified, commodity x86 hardware, defenses based on data-flow integrity may double the memory requirements, and may make execution up to 37 times slower [32]. Because these defenses also double the number of memory accesses, even the most heavily optimized mechanism is still likely to run software twice as slow [9]. Such overheads are likely to be unacceptable in many scenarios, e.g., for server workloads where a proportional increase in cost may be expected. Therefore, in practice, these defenses may never see widespread adoption - especially since equally good protection may be achievable using a combination of the below defenses.

This section does not attempt a comprehensive survey of the literature on these defenses. The survey by Younan, Joosen and Piessens provides an overview of the state of the art of countermeasures for the types of attacks discussed in this chapter[41, 42]

\subsection{Defense 1: Checking Stack Canaries on Return Addresses}

The $\mathrm{C}$ and $\mathrm{C}++$ languages do not specify how function return addresses are represented in stack memory. Rather, these, and many other programming languages, hold abstract most elements of a function's invocation stack frame in order to allow for portability between hardware architectures and to give compilers flexibility in choosing an efficient low-level representation. This flexibility enables an effective defense against some attacks, such as the return-address clobbering of Attack 1.

In particular, on function calls, instead of storing return addresses directly onto the stack, $\mathrm{C}$ and $\mathrm{C}++$ compilers are free to generate code that stores return addresses in an encrypted and signed form, using a local, secret key. Then, before each function return, the compiler could emit code to decrypt and validate the integrity of the return address about to be used. In this case, assuming that strong cryptography is used, an attacker that did not know the key would be unable to cause the target software to return to an address of their choice as a result of a stack corruption - even when the target software contains an exploitable buffer overflow vulnerability that allows such corruption.

In practice, it is desirable to implement an approximation of the above defense, and get most of the benefits without incurring the overwhelming cost of executing cryptography code on each function call and return. 


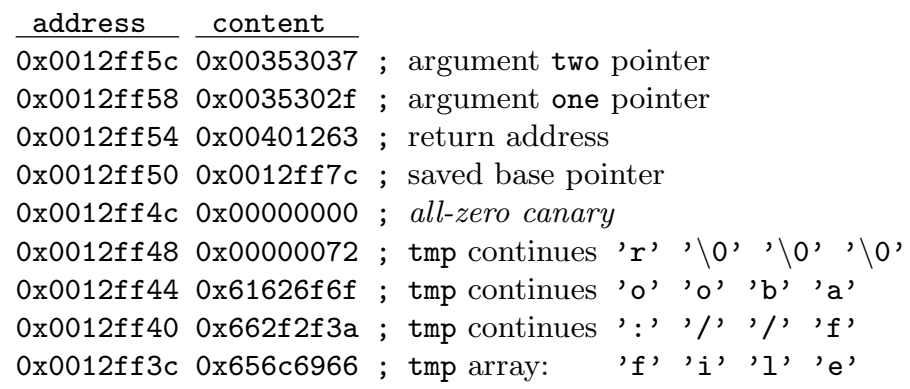

Fig. 16. A stack snapshot like that shown in Fig. 4 where a "canary value" has been placed between the tmp array and the saved base pointer and return address. Before returning from functions with vulnerabilities like those in Attack 1, it is an effective defense to check that the canary is still zero: an overflow of a zero-terminated string across the canary's stack location will not leave the canary as zero

One such approximation requires no secret, but places a public canary value right above function-local stack buffers. This value is designed to warn of dangerous stack corruption, much as a coal-mine canary would warn about dangerous air conditions. Figure 16 shows an example of a stack with an all-zero canary value. Validating the integrity of this canary is an effective means of ensuring that the saved base pointer and function return address have not been corruptedgiven the assumption that attacks are only possible through stack corruption based on the overflow of a string buffer. For improved defenses, this public canary may contain other bytes, such as newline characters, that frequently terminate the copying responsible for string-based buffer overflows. For example, some implementations have used the value 0x000aff0d as the canary [13].

Stack-canary defenses may be improved by including in the canary value some bits that should be unknown to the attacker. For instance, this may help defend against return-address clobbering with an integer overflow, such as is enabled by the memcpy vulnerability in Fig. 9. Therefore, some implementations of stack canary defenses, such as Microsoft's /GS compiler option [7], are based on a random value, or cookie.

Figure 17 shows the machine code for a function compiled with Microsoft's /GS option. The function preamble and postamble each have three new instructions that set and check the canary, respectively. With /GS, the canary placed on the stack is a combination of the function's base pointer and the function's module cookie. Module cookies are generated dynamically for each process, using good sources of randomness (although some of those sources are observable to an attacker running code on the same system). Separate, fresh module cookies are used for the executable and each dynamic library within a process address space (each has its own copy of the __security_cookie variable in Fig. 17). As a result, in a stack with multiple canary values, each will be unique, with more dissimilarity where the stack crosses module boundaries. 


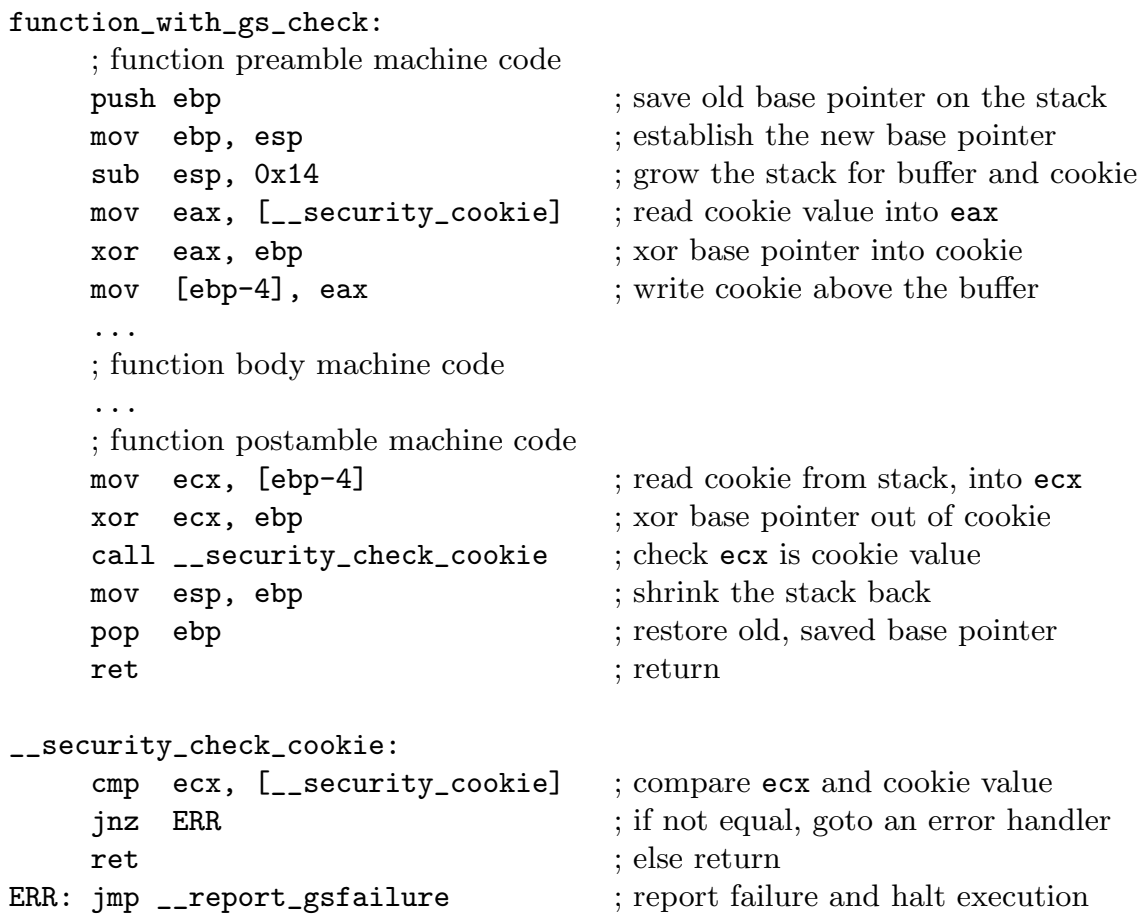

Fig. 17. The machine code for a function with a local array in a fixed-size, 16-byte stack buffer, when compiled using the Windows / GS implementation of stack cookies in the most recent version of the Microsoft $\mathrm{C}$ compiler [7,22]. The canary is a random cookie value, combined with the base pointer. In case the local stack buffer is overflowed, this canary is placed on the stack above the stack buffer, just below the return address and saved base pointer, and checked before either of those values are used

\section{Defense 1: Overhead, Limitations, Variants, and Counterattacks}

There is little enforcement overhead from stack canary defenses, since they are only required in functions with local stack buffers that may be overflowed. (An overflow in a function does not affect the invocation stack frames of functions it calls, which are lower on the stack; that function's canary will be checked before any use of stack frames that are higher on the stack, and which may have been corrupted by the overflow.) For most $\mathrm{C}$ and $\mathrm{C}++$ software this overhead amounts to a few percent $[13,15]$. Even so, most implementations aim to reduce this overhead even further, by only initializing and checking stack canaries in functions that contain a local string char array, or meet other heuristic requirements. As a result, this defense is not always applied where it might be useful - as evidenced by the recent ANI vulnerability in Windows [20].

Stack canaries can be an efficient and effective defense against Attack 1, where the attacker corrupts function-invocation control data on the stack. However, stack canaries only check for corruption at function exit. Thus, they offer no 
defense against Attacks 2, 3, and 4, which are based on corruption of the heap, function-pointer arguments, or global data pointers.

Stack canaries are a widely deployed defense mechanism. In addition to Microsoft's /GS, StackGuard [13] and ProPolice [15] are two other notable implementations. Given its simple nature, it is somewhat surprising that there is significant variation between the implementations of this defense, and these implementations have varied over time $[7,19]$. This reflects the ongoing arms race between attackers and defenders.

Stack canary defenses are subject to a a number of counterattacks. Most notably, even when the only exploitable vulnerability is a stack-based buffer overflow, the attackers may be able to craft an attack that is not based on return-address clobbering. For example, the attack may corrupt a local variable, an argument, or some other value that is used before the function exits.

Also, the attacker may attempt to guess, or learn the cookie values, which can lead to a successful attack given enough luck or determination. The success of this counterattack will depend on the exploited vulnerability, the attacker's access to the target system, and the particulars of the target software. (For example, if stack canaries are based on random cookies, then the attacker may be able to exploit certain format-string vulnerabilities to learn which canary values to embed in the data of the buffer overflow.)

Due to the counterattack where attackers overwrite a local variable other than the return address, most implementations have been extended to reorder organization of the stack-frame.

Most details about the function-invocation stack frame are left unspecified in the $\mathrm{C}$ and $\mathrm{C}++$ languages, to give flexibility in the compilation of those language aspects down to a low-level representation. In particular, the compiler is free to lay out function-local variables in any order on the stack, and to generate code that operates not on function arguments, but on copies of those arguments.

This is the basis of the variant of this countermeasure. In this defense, the compiler places arrays and other function-local buffers above all other functionlocal variables on the stack. Also, the compiler makes copies of function arguments into new, function-local variables that also sit below any buffers in the function. As a result, these variables and arguments are not subject to corruption through an overflow of those buffers.

The stack cookie will also provide detection of attacks that try to overwrite data of previous stack frames. Besides the guessing attack described earlier, two counterattacks still exist to this extended defense. In a first attack, an attacker can still overwrite the contents of other buffers that may be stored above the buffer that overflows. A second attack occurs when an attacker overwrite information of any other stack frames or other information that is stored above the current stack frame. If this information is used before the current function returns (i.e., before the cookie is checked), then an attack may be possible. An example of such an attack was possible against [7]: an attacker would overwrite the exception-handler pointers, which is stored on the stack above the function stack frames. The attacker would then cause an exception (e.g., a stack overflow 
exception or a cookie mismatch exception), which would result in the attacker's code being executed [29]. This specific attack was countered by applying Defense 3 to the exception handler.

\subsection{Defense 2: Making data not be executable as machine code}

Many high-level languages allow code and data to reside in two, distinct types of memory. The $\mathrm{C}$ and $\mathrm{C}++$ languages follow this tradition, and do not specify what happens when code pointers are read and written as data, or what happens when a data pointer is invoked as if it were a function pointer. This underspecification brings important benefits to the portability of $\mathrm{C}$ and $\mathrm{C}++$ software, since it must sometimes run on systems where code and data memory are truly different. It also enables a particularly simple and efficient defense against directcode-injection exploits, such as those in Attacks 1 and 2.

If data memory is not executable, then Attacks 1 and 2 fail as soon as the hardware instruction pointer reaches the first byte of the attack payload (e.g., the bytes 0xfeeb2ecd described in Fig. 6, and used throughout this chapter). Even when the attacker manages to control the flow of execution, they cannot simply make control proceed directly to their attack payload. This is a simple, useful barrier to attack, which can be directly applied to most software, since, in practice, most software never treats data as code.

(Some legacy software will execute data as a matter of course; other software uses self-modifying code and writes to code memory as a part of regular, valid execution. For example, this behavior can be seen in some efficient, just-in-time interpreters. However, such software can be treated as a special case, since it is uncommon and increasingly rare.)

\section{Defense 2: Overhead, Limitations, Variants, and Counterattacks}

In its implementation on modern x86 systems, non-executable data has some performance impact because it relies on double-size, extended page tables. The NX page-table-entry bit, which flags memory as non-executable, is only found in PAE page tables, which are double the size of normal tables, and are otherwise not commonly used. The precise details of page-table entries can significantly impact the overall system performance, since page tables are a frequently-consulted part of the memory hierarchy - with thousands of lookups a second and, in some cases, a lookup every few instructions. However, for most workloads, the overhead should be in the small percents, and will often be close to zero.

Non-executable data defends against direct code injection attacks, but offers no barrier to exploits such as those in Attacks 3 and 4. For any given direct codeinjection attack, it is likely that an attacker can craft an indirect jump-to-libc variant, or a data-only exploit [10]. Thus - although this defense can be highly useful when used in combination with other defenses - by itself, it is not much of a stumbling block for attackers.

On Microsoft Windows, and most other platforms, software will typically execute in a mode where writing to code memory generates a hardware exception. 
In the past, some systems have also generated such an exception when the hardware instruction pointer is directed to data memory, i.e., upon an attempt to execute data as code. However, until recently, commodity x 86 hardware has only supported such exceptions through the use of segmented memory - which runs counter to the flat memory model that is fundamental to most modern operating systems. (Despite being awkward, x86 segments have been used to implement non-executable memory, e.g., stacks, but these implementations are limited, for instance in their support for multi-threading and dynamic libraries.)

Since 2003, and Windows XP SP2, commodity operating systems have come to support the $\mathrm{x} 86$ extended page tables where any given memory page may be marked as non-executable, and x86 vendors have shipped processors with the required hardware support. Thus, it is now the norm for data memory to be non-executable.

Indirect code injection, jump-to-libc attacks, and data-only attacks are all effective counterattacks to this defense Even so, non-executable data can play a key role in an overall defense strategy; for instance, when combined with Defense 4 below, this defense can prevent an attacker from knowing the location of any executable memory bytes that could be useful to an attack.

\subsection{Defense 3: Enforcing control-flow integrity on code execution}

As in all high-level languages, it is not possible for software written in the $\mathrm{C}$ and $\mathrm{C}++$ languages to perform arbitrary control-flow transfers between any two points in its code. Compared to the exclusion of data from being executed as code, the policies on control-flow between code are much more fine-grained

For example, the behavior of function calls is only defined when the callee code is the start of a function - even when the caller invokes that code through a function pointer. Also, it is not valid to place a label into an expression, and goto to that label, or otherwise transfer control into the middle of an expression being evaluated. Transferring control into the middle of a machine code instruction is certainly not a valid, defined operation, in any high-level language - even though the hardware may allow this, and this may be useful to an attacker (see Attack 3, page 14).

Furthermore, within the control flow that a language permits in general, only a small fraction will, in fact, be possible in the semantics of a particular piece of software written in that language. For most software, control flow is either completely static (e.g., as in a C goto statement), or allows only a small number of possibilities during execution.

Similarly, for all C or C++ software, any indirect control transfers, such as through function pointers or at return statements, will have only a small number of valid targets.

Dynamic checks can ensure that the execution of low-level software does not stray from a restricted set of possibilities allowed by the high-level software. The runtime enforcement of such a Control-Flow Integrity, or CFI, security policy is a highly effective defense against low-level software attacks $[1,2]$. 


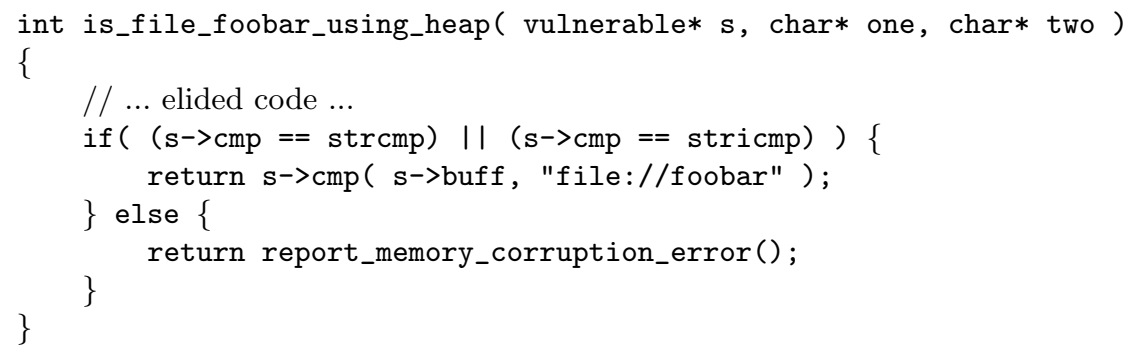

Fig. 18. An excerpt of the $\mathrm{C}$ code in Fig. 7 with explicit CFI checks that only allow the proper comparison methods to be invoked at runtime - assuming only strcmp and stricmp are possible. These CFI checks prevent the exploit on this function in Attack 2
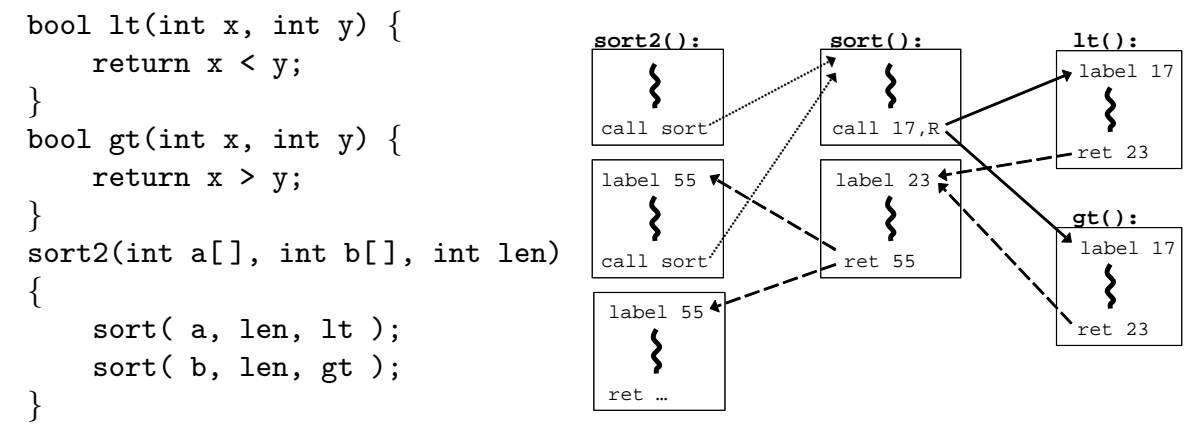

Fig. 19. Three $\mathrm{C}$ functions and an outline of their possible control flow, as well as how an CFI enforcement mechanism based on CFI labels might apply to the functions. In the outline, the CFI labels 55, 17, and 23 are found at the valid destinations of computed control-flow instructions; each such instruction is also annotated with a CFI label that corresponds to its valid destinations

There are several strategies possible in the implementation of CFI enforcement. For instance, CFI may be enforced by dynamic checks that compare the target address of each computed control-flow transfer to a set of allowed destination addresses. Such a comparison may be performed by the machine-code equivalent of a switch statement over a set of constant addresses. Programmers can even make CFI checks explicitly in their software, as shown in Fig. 18. However, unlike in Fig. 18, it is not possible to write software that explicitly performs CFI checks on return addresses, or other inaccessible pointers; for these, CFI checks must be added by the compiler, or some other mechanism. Also, since the set of allowed destination addresses may be large, any such sequence of explicit comparisons is likely to lead to unacceptable overhead.

One efficient CFI enforcement mechanism, described in [1], modifies according to a given control-flow graph, both the source and destination instructions of computed control-flow transfers. Two destinations are equivalent, when the CFG contains edges to each from the same set of sources. At each destination, a CFI 


\begin{tabular}{|c|c|c|c|c|c|}
\hline \\
\hline \multicolumn{6}{|l|}{$0 \times 57$} \\
\hline \multicolumn{6}{|c|}{$0 \times 53$} \\
\hline $0 \times 8 b$ & $0 \times 44$ & $0 \times 240 \times 24$ & & & \\
\hline $0 \times 81$ & $0 \times 78$ & $0 \times f c \quad 0 \times 78$ & $0 \times 56$ & $0 \times 34$ & $0 \times 12$ \\
\hline $0 \times 75$ & $0 \times 13$ & & & & \\
\hline Oxff & $0 x d 0$ & & & & \\
\hline $0 x 0 f$ & $0 \times 18$ & 0x80 0xdd & $0 x c c$ & $0 x b b$ & Oxaa \\
\hline $0 \times 83$ & $0 x c 4$ & $0 \times 08$ & & & \\
\hline $0 \times 85$ & $0 \mathrm{xcO}$ & & & & \\
\hline $0 \times 7 e$ & $0 \times 02$ & & & & \\
\hline
\end{tabular}

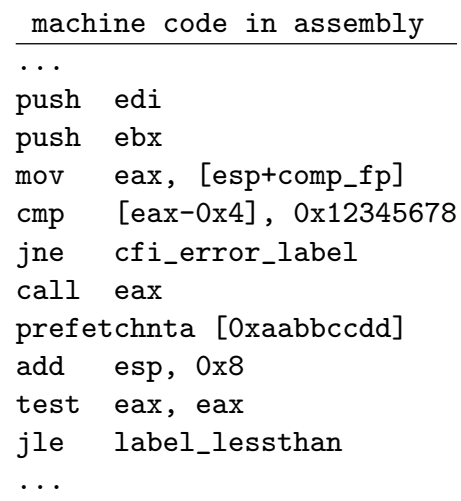

Fig. 20. A version of Fig. 10, showing how CFI checks as in [1] can be added to the qsort library function where it calls the comparison function pointer. Before calling the pointer, it is placed in a register eax, and a comparison establishes that the four bytes $0 \times 12345678$ are found immediately before the destination code, otherwise execution goes to a security error. After the call instruction, an executable, side-effect-free instruction embeds the constant 0xaabbccdd; by comparing against this constant, the comparison function can establish that it is returning to a valid call site

label is inserted, that identifies equivalent destinations, i.e., destinations with the same set of possible sources. The CFI labels embed a value, or bit pattern, that distinguishes each; these values need not be secret. Before each source instruction, a dynamic CFI check is inserted that ensures that the runtime destination has the proper CFI label.

Fig. 19 shows a $\mathrm{C}$ program fragment demonstrating this CFI enforcement mechanism. In this figure, a function sort2 calls a qsort-like function sort twice, first with $1 \mathrm{t}$ and then with gt as the pointer to the comparison function. The right side of Fig. 19 shows an outline of the machine-code blocks for these four functions and all control-flow-graph edges between them. In the figure, edges for direct calls are drawn as light, dotted arrows; edges from source instructions are drawn as solid arrows, and return edges as dashed arrows. In this example, sort can return to two different places in sort2. Therefore, there are two CFI labels in the body of sort2, and an CFI check when returning from sort, using 55 as the CFI label. (Note that CFI enforcement does not guarantee to which of the two callsites sort must return; for this, other defenses, such as Defense 1, must be employed.)

Also, in Fig. 19, because sort can call either lt or gt, both comparison functions start with the CFI label 17, and the call instruction, which uses a function pointer in register $\mathrm{R}$, performs a CFI check for 17. Finally, the CFI label 23 identifies the block that follows the comparison callsite in sort, so both comparison functions return with a CFI check for 23.

Figure 20 shows a concrete example of how CFI enforcement based on CFI labels can look, in the case of x86 machine-code software. Here, the CFI label 0x12345678 identifies all comparison routines that may be invoked by qsort, 
and the CFI label Oxaabbccdd identifies all of their valid call sites. This style of CFI enforcement has good performance, and also gives strong guarantees. By choosing the bytes of CFI labels carefully, so they don't overlap with code, even an attacker that controls all of data memory cannot divert execution from the permitted control-flow graph - assuming that data is also non-executable.

The CFI security policy dictates that software execution must follow a path of a control-flow graph, determined ahead of time, that represents all possible valid executions of the software. This graph can be defined by analysis - source-code analysis, binary analysis, or execution profiling. This graph does not need to be perfectly accurate, but needs only be a conservative approximation of the controlflow graph possible in the software, as written in its high-level programming language. To be conservative, the graph must err on the side of allowing all valid executions of the software, even this may entail allowing some invalid executions as well. For instance, the graph might conservatively permit the start of a few-

too-many functions as the valid destinations of a source instruction where a function pointer is invoked.

\section{Defense 3: Overhead, Limitations, Variants, and Counterattacks}

CFI enforcement incurs only modest overhead. With the CFI enforcement mechanism in [1], which instruments x86 machine code much as is shown in Fig. 20, the reported code-size increase is around $8 \%$, and execution slowdown ranges from $0 \%$ to $45 \%$ on a set of processor benchmarks, with a mean of $16 \%$ Even so, this overhead is significant enough that CFI enforcement has, to date, seen only limited adoption. However, a form of CFI is enforced by the Windows SafeSEH mechanism, which limits dispatching of exceptions to a set of statically-declared exception handlers; this mechanism does not incur measurable overheads.

CFI enforcement offers no protection against Attack 4 or other data-only attacks. However, CFI can be an highly effective defense against all attacks based on controlling machine-code execution, including Attacks 1, 2, and 3 .

In particular, CFI enforcement is likely to prevent all variants of Attack 3, i.e., jump-to-libc attacks that employ trampolines or opportunistic executable byte sequences such as those found embedded within machine-code instructions. This is the case even if CFI enforces only a coarse-grained approximation of the software control-flow graph, such as allowing function-pointer calls to the start of any function with the same argument types, and allowing functions to return to any of their possible call sites [1].

CFI enforcement mechanisms vary both in their mechanisms and in their policy. Some mechanisms establish the validity of each computed control transfer by querying a separate, static data structure, which can be a hash table, a bit vector, or a structure similar to multi-level page tables [38]. Other mechanisms execute the software in a fast machine-code interpreter that enforces CFI on control flow [26]. Finally, a coarse-grained form of CFI can be enforced by making all computed-control-flow destinations be aligned on multi-word boundaries. (However, in this last case, any "basic block" is effectively a valid destination, so trampolines and elaborate jump-to-libc attacks are still feasible.) The com- 
plexity and overheads of these CFI mechanisms varies, but is typically greater than that described above, based on CFI labels.

In a system with CFI enforcement, any exploit that does not involve controlling machine-code execution is a likely counterattack; this includes not only data-only attacks, such as Attack 4, but also other, higher-level attacks, such as social engineering and flaws in programming interfaces [4]. In addition, depending on the granularity of CFI enforcement policy, and how it is used in combination with other defenses, there may still exist possibilities for certain jump-to-libc attacks, for instance where a function is made to return to a dynamically-incorrect, but statically-possible, call site.

\subsection{Defense 4: Randomizing the layout of code and data in memory}

The $\mathrm{C}$ and $\mathrm{C}++$ languages specify neither where code is located in memory, nor the location of variables, arrays, structures, or objects. For software compiled from these languages, the layout of code and data in memory is decided by the compiler and execution environment. This layout directly determines all concrete addresses used during execution - and attacks, including all of the attacks in Sect. 2, typically depend on these concrete addresses.

Therefore, a simple, pervasive form of address encryption can be achieved by shuffling, or randomizing, the layout of software in the memory address space, in a manner that is unknown to the attacker. Defenses based on such AddressSpace Layout Randomization, or ASLR, can be a highly practical, effective barrier against low-level attacks. Such defenses were first implemented in the PaX project [33] and have recently been deployed in Windows Vista [18, 22].

ASLR defenses can be used to change the addresses of all code, global variables, stack variables, arrays, and structures, objects, and heap allocations; with ASLR those addresses are derived from a random value, chosen for the software being executed and the system on which it executes. These addresses, and the memory-layout shuffling, may be public information on the system where the software executes. However, low-level software attacks - including most worms, viruses, adware, spyware, and malware - are often performed by remote attackers that have no existing means of running code on their target system, or otherwise inspect the addresses utilized on that system. To overcome ASLR defenses, such attackers will have to craft attacks that do not depend on addresses, or somehow guess or learn those addresses.

ASLR is not intended to defend against attackers that are able to control the software execution, even to a very small degree. Like many other defenses that rely on secrets, ASLR is easily circumvented by an attacker that can read the software's memory. Once an attacker is able to execute even the smallest amount of code of their choice (e.g., in a jump-to-libc attack), it should be safely assumed that the attacker can read memory and, in particular, that ASLR is no longer an obstacle. Fortunately, ASLR and the other defenses in this chapter can be highly effective in preventing attackers from successfully executing even a single machine-code instruction of their choice. 


\begin{tabular}{|c|c|c|c|c|}
\hline stack & one & stack & two & \\
\hline address & contents & address & contents & \\
\hline$\overline{0 x 0022 f e a c}$ & $\overline{0 x 008 a 13 e 0}$ & $\overline{0 \times 0013 f 750}$ & $\overline{0 \mathrm{x} 00 \mathrm{~b} 113 \mathrm{e} 0}$ & ; cmp argument \\
\hline 0x0022fea8 & $0 \times 00000001$ & $0 \times 0013 f 74 c$ & $0 \times 00000001$ & ; len argument \\
\hline $0 \times 0022$ fea 4 & $0 x 00 a 91147$ & $0 \times 0013 f 748$ & $0 x 00191147$ & ; data argument \\
\hline 0x0022fea0 & $0 \times 008 a 1528$ & $0 \times 0013 f 744$ & 0x00b11528 & ; return address \\
\hline $0 x 0022 f e 9 c$ & $0 x 0022 f e c 8$ & $0 \times 0013 f 740$ & $0 x 0013 f 76 c$ & ; saved base pointer \\
\hline $0 x 0022 f e 98$ & $0 \times 00000000$ & $0 x 0013 f 73 c$ & $0 \times 00000000$ & ; tmp final 4 bytes \\
\hline 0x0022fe94 & $0 \times 00000000$ & $0 \times 0013 f 738$ & $0 \mathrm{x} 00000000$ & ; tmp continues \\
\hline $0 \times 0022 f e 90$ & $0 \times 00000000$ & $0 \times 0013 f 734$ & $0 \times 00000000$ & ; tmp continues \\
\hline $0 x 0022 f e 8 c$ & $0 \times 00000000$ & $0 \times 0013 f 730$ & $0 \mathrm{x} 00000000$ & ; tmp continues \\
\hline $0 x 0022 f e 88$ & $0 \times 00000000$ & $0 x 0013 f 72 c$ & $0 \times 00000000$ & ; tmp continues \\
\hline $0 x 0022$ fe84 & $0 \times 00000000$ & $0 x 0013 f 728$ & $0 \times 00000000$ & ; tmp continues \\
\hline $0 x 0022 f e 80$ & $0 \times 00000000$ & $0 \times 0013 f 724$ & $0 \times 00000000$ & ; tmp continues \\
\hline $0 x 0022 f e 7 c$ & $0 \times 00000000$ & $0 \times 0013 f 720$ & $0 \times 00000000$ & ; tmp buffer starts \\
\hline $0 x 0022 f e 78$ & $0 \times 00000004$ & $0 x 0013 f 71 c$ & $0 \times 00000004$ & ; memcpy length argument \\
\hline $0 \times 0022 f e 74$ & $0 x 00 a 91147$ & $0 x 0013 f 718$ & $0 x 00191147$ & ; memcpy source argument \\
\hline $0 \times 0022 f e 70$ & $0 x 0022 f e 8 c$ & $0 x 0013 f 714$ & $0 \times 0013 f 730$ & ; memcpy destination arg. \\
\hline
\end{tabular}

Fig. 21. The addresses and contents of the stacks of two different executions of the same software, given the same input. The software is the median function of Fig. 9, the input is an array of the single integer zero, and the stacks are snapshots taken at the same point as in Fig. 12. The snapshots are taken from two executions of that function on Windows Vista, with a system restart between the executions. As a result of ASLR defenses, only the input data remains the same in the two executions. All addresses are different; even so, some address bits remain the same since, for efficiency and compatibility with existing software, ASLR is applied only at a coarse granularity

As a concrete example of ASLR, Fig. 21 shows two execution stacks for the median function of Fig. 9, taken from two executions of that function on Windows Vista, which implements ASLR defenses. These stacks contain code addresses, including a function pointer and return address; they also include addresses in data pointers that point into the stack, and in the data argument which points into the heap. All of these addresses are different in the two executions; only the integer inputs remain the same.

On many software platforms, ASLR can be applied automatically, in manner that is compatible even with legacy software. In particular, ASLR changes only the concrete values of addresses, not how those addresses are encoded in pointers; this makes ASLR compatible with common, legacy programming practices that depend on the encoding of addresses.

However, ASLR is both easier to implement, and is more compatible with legacy software, when data and code is shuffled at a rather coarse granularity. For instance, software may simultaneously use more than a million heap allocations; however, on a 32-bit system, if an ASLR mechanism randomly spread those allocations uniformly throughout the address space, then only small contiguous memory regions would remain free. Then, if that software tried to allocate an array whose size is a few tens of kilobytes, that allocation would most 
likely fail - even though, without this ASLR mechanism, it might certainly have succeeded. On the other hand, without causing incompatibility with legacy software, an ASLR mechanism could change the base address of all heap allocations, and otherwise leave the heap implementation unchanged. (This also avoids triggering latent bugs, such as the software's continued use of heap memory after deallocation, which are another potential source of incompatibility.)

In the implementation of ASLR on Windows Vista, the compilers and the execution environment have been modified to avoid obstacles faced by other implementations, such as those in the PaX project [33]. In particular, the software executables and libraries of all operating system components and utilities have been compiled with information that allows their relocation in memory at load time. When the operating system starts, the system libraries are located sequentially in memory, in the order they are needed, at a starting point chosen randomly from 256 possibilities; thus a jump-to-libc attack that targets the concrete address of a library function will have less than a $0.5 \%$ chance of succeeding. This randomization of system libraries applies to all software that executes on the Vista operating system; the next time the system restarts, the libraries are located from a new random starting point.

When a Windows Vista process is launched, several other addresses are chosen randomly for that process instance, if the main executable opts in to ASLR defenses. For instance, the base of the initial heap is chosen from 32 possibilities. The stacks of process threads are randomized further: the stack base is chosen from 32 possibilities, and a pad of unused memory, whose size is random, is placed on top of the stack, for a total of about 16 thousand possibilities for the address of the initial stack frame. In addition, the location of some other memory regions is also chosen randomly from 32 possibilities, including thread control data and the process environment data (which includes the table corrupted in Attack 4). For processes, the ASLR implementation chooses new random starting points each time that a process instance is launched.

An ASLR implementation could be designed to shuffle the memory layout at a finer granularity than is done in Windows Vista. For instance, a pad of unused memory could be inserted within the stack frame of all (or some) functions; also, the inner memory allocation strategy of the heap could be randomized. However, in Windows Vista, such an ASLR implementation would incur greater overhead, would cause more software compatibility issues, and might be likely to thwart mostly attacks that are already covered by other deployed defenses. In particular, there can be little to gain from shuffling the system libraries independently for each process instance [37] - and such an ASLR implementation would be certain to cause large performance and resource overheads.

\section{Defense 4: Overhead, Limitations, Variants, and Counterattacks}

The enforcement overhead of ASLR defenses will vary greatly depending on the implementation. In particular, implementations where shared libraries may be placed at different addresses in different processes will incur greater overhead and consume more memory resources. 
However, in its Windows Vista implementation, ASLR may actually slightly improve performance. This improvement is a result of ASLR causing library code to be placed contiguously into the address space, in the order that the code is actually used. This encourages a tight packing of frequently-used page table entries, which has performance benefits (cf., the page-table changes for non-executable data, discussed on page 22).

ASLR can provide effective defenses against all of the attacks in Sect. 2 of this chapter, because it applies to the addresses of both code and data. Even so, some data-only attacks remain possible, where the attacks do not depend on concrete addresses, but rely on corrupting the contents of the data being processed by the target software.

The more serious limitation of ASLR is the small number of memory layout shuffles that are possible on commodity 32-bit hardware - especially given the coarse shuffling granularity that is required for efficiency and compatibility with existing software. As a result, ASLR creates only at most a few thousand possibilities that an attacker must consider, and any given attack will be successful against a significant (albeit small) number of target systems. The number of possible shuffles in an ASLR implementation can be greatly increased on 64-bit platforms, which are starting to be adopted. However, current 64-bit hardware is limited to 48 usable bits and can therefore offer at most a 64 -thousand-fold increase in the number of shuffles possible [40].

Furthermore, at least on 32-bit systems, the number of possible ASLR shuffles is insufficient to provide a defense against scenarios where the attacker is able to retry their attack repeatedly, with new addresses [37]. Such attacks are realistic. For example, because a failed attack did not crash the software in the case of the recent ANI vulnerability in Windows [20], an attack, such as a script in a malicious Web page, could try multiple addresses until a successful exploit was found. However, in the normal case, when failed attacks crash the target software, attacks based on retrying can be mitigated by limiting the number of times the software is restarted. In the ASLR implementation in Windows Vista, such limits are in place for many system components.

ASLR defenses provide one form of software diversity, which has been long known to provide security benefits. One way to achieve software diversity is to deploy multiple, different implementations of the same functionality. However, this approach is costly and may offer limited benefits: its total cost is proportional to the number of implementations and programmers are known to make the same mistakes when implementing the same functionality [30].

ASLR has a few counterattacks other than the data-only, content-based attacks, and the persistent guessing of an attacker, which are both discussed above. In particular, an otherwise harmless information-disclosure vulnerability may allow an attacker to learn how addresses are shuffled, and circumvent ASLR defenses. Although unlikely, such a vulnerability may be present because of a format-string bug, or because the contents of uninitialized memory are sent on the network when that memory contains residual addresses. 
Another type of counterattack to ASLR defenses is based on overwriting only the low-order bits of addresses, which are predictable because ASLR is applied at a coarse granularity. Such overwrites are sometimes possible through buffer overflows on little-endian architectures, such as the x86. For example, in Fig. 21, if there were useful trampoline machine-code to be found seven bytes into the cmp function, then changing the least-significant byte of the cmp address on the stack from 0xe0 to 0xe7 would cause that code to be invoked. An attacker that succeeded in such corruption might well be able to perform a jump-tolibc attack much like that in Attack 3. (However, for this particular stack, the attacker would not succeed, since the cmp address will always be overwritten completely when the vulnerability in the median function in Fig. 9 is exploited.)

Despite the above counterattacks, ASLR is an effective barrier to attack, especially when combined with the defenses described previously in this section.

\section{Summary and discussion}

The distinguishing characteristic of low-level software attacks is that they are dependent on the low-level details of the software's executable representation and its execution environment. As a result, defenses against such attacks can be based on changing those details in ways that are compatible with the software's specification in a higher-level programming language.

As in Defense 1, integrity bits can be added to the low-level representation of state, to make attacks more likely to be detected, and stopped. As in Defenses 2 and 3, the low-level representation can be augmented with a conservative model of behavior and with runtime checks that ensure execution conforms to that model. Finally, as in Defenses 1 and 4, the low-level representation can be encoded with a secret that the attacker must guess, or otherwise learn, in order to craft functional attacks.

However, defenses like those in this chapter fall far short of a guarantee that the software exhibits only the low-level behavior that is possible in the software's higher-level specification. Such guarantees are hard to come by. For languages like $\mathrm{C}$ and $\mathrm{C}++$, there are efforts to build certifying compilers that can provide such guarantees, for correct software [6,28]. Unfortunately, even these compilers offer few, or no guarantees in the presence of bugs, such as buffer-overflow vulnerabilities. Some compiler techniques, such as bounds checking, can reduce or eliminate the problem of buffer-overflow vulnerabilities. However, due to the existence of programmer-manipulated pointers, applying such checks to $\mathrm{C}$ is a hard problem. As a result, this type of checking comes at a hefty cost to performance, lacks scalability or results in code incompatibility [24]. While recently advances have been made with respect to performance and compatibility, these newer approaches still suffer from scalability problems [14], or achieve higher performance by being less accurate [3]. These problems are the main reasons that this type of checking has not made it into mainstream operating systems and compilers. 


\begin{tabular}{|c|c|c|c|c|}
\hline & $\begin{array}{l}\text { Return } \\
\text { address } \\
\text { corruption } \\
\text { (A1) }\end{array}$ & $\begin{array}{l}\text { Heap } \\
\text { function } \\
\text { pointer } \\
\text { corruption } \\
\text { (A2) }\end{array}$ & $\begin{array}{l}\text { Jump-to- } \\
\text { libc (A3) }\end{array}$ & $\begin{array}{l}\text { Non- } \\
\text { control } \\
\text { data (A4) }\end{array}$ \\
\hline Stack Canary (D1) & $\begin{array}{l}\text { Partial } \\
\text { defense }\end{array}$ & & $\begin{array}{l}\text { Partial } \\
\text { defense }\end{array}$ & $\begin{array}{l}\text { Partial } \\
\text { defense }\end{array}$ \\
\hline $\begin{array}{l}\text { Non-executable data } \\
\text { (D2) }\end{array}$ & $\begin{array}{l}\text { Partial } \\
\text { defense }\end{array}$ & $\begin{array}{l}\text { Partial } \\
\text { defense }\end{array}$ & $\begin{array}{l}\text { Partial } \\
\text { defense }\end{array}$ & \\
\hline $\begin{array}{l}\text { Control-flow integrity } \\
\text { (D3) }\end{array}$ & $\begin{array}{l}\text { Partial } \\
\text { defense }\end{array}$ & $\begin{array}{l}\text { Partial } \\
\text { defense }\end{array}$ & $\begin{array}{l}\text { Partial } \\
\text { defense }\end{array}$ & \\
\hline $\begin{array}{l}\text { Address space layout } \\
\text { randomization (D4) }\end{array}$ & $\begin{array}{l}\text { Partial } \\
\text { defense }\end{array}$ & $\begin{array}{l}\text { Partial } \\
\text { defense }\end{array}$ & $\begin{array}{l}\text { Partial } \\
\text { defense }\end{array}$ & $\begin{array}{l}\text { Partial } \\
\text { defense }\end{array}$ \\
\hline
\end{tabular}

Table 1. A table of the relationship between the attacks and defenses in this chapter. None of the defenses completely prevent the attacks, in all of their variants. The first defense applies only to the stack, and is not an obstacle to the heap-based Attack 2 . Defenses 2 and 3 apply only to the control flow of machine-code execution, and do not prevent the data-only Attack 4. When combined with each other, the defenses are stronger than when they are applied in isolation

Many of the bugs can be eliminated by using other, advanced compiler techniques, like those used in the Cyclone [23], CCured [31], and Deputy [43] systems. But these techniques are not widely applicable: they require pervasive source-code changes, runtime memory-management support, restrictions on concurrency, and result in significant enforcement overhead.

In comparison, the defenses in this chapter have very low overheads, require no source code changes but at most re-compilation, and are widely applicable to legacy software written in $\mathrm{C}, \mathrm{C}++$, and similar languages. For instance, they have been applied pervasively to recent Microsoft software, including all the components of the Windows Vista operating system. As in that case, these defenses are best used as one part of a comprehensive software-engineering methodology designed to to reduce security vulnerabilities. Such a methodology should include, at least, threat analysis, design and code reviews for security, security testing, automatic analysis for vulnerabilities, and the rewriting of software to use safer languages, interfaces, and programming practices [21].

The combination of the defenses in this chapter forms a substantial, effective barrier to all low-level attacks - although, as summarized in Table 1, each offers only partial protection against certain attacks. In particular, they greatly reduce the likelihood that an attacker can exploit a low-level security vulnerability for purposes other than a denial-of-service attack. The adoption of these countermeasures, along with continuing research in this area which further improves the protection offered by such countermeasures and with improved programming 
practices which aim to eliminate buffer overflows and other underlying security vulnerabilities, offers some hope that, for $\mathrm{C}$ and $\mathrm{C}++$ software, low-level software security may become less of a concern in the future.

Acknowledgments Thanks to Martín Abadi for suggesting the structure of the original tutorial, and to Yinglian Xie for proofreading and for suggesting useful improvements to the exposition.

\section{References}

1. M. Abadi, M. Budiu, Ú. Erlingsson, and J. Ligatti. Control-flow integrity. In Proceedings of the 12th ACM Conference on Computer and Communications Security (CCS'05), pages 340-353, Alexandria, VA, USA, November 2005. ACM.

2. M. Abadi, M. Budiu, Ú. Erlingsson, and J. Ligatti. A theory of secure control flow. In Proceedings of the 7th International Conference on Formal Engineering Methods (ICFEM'05), Manchester, UK, November 2005.

3. P. Akritidis, C. Cadar, C. Raiciu, M. Costa, and M. Castro. Preventing memory error exploits with WIT. In Proceedings of the 2008 IEEE Symposium on Security and Privacy, Oakland, CA, USA, May 2008. IEEE.

4. R.J. Anderson. Security Engineering: A Guide to Building Dependable Distributed Systems. John Wiley \& Sons, Inc., New York, NY, USA, 2001.

5. M. Bailey, E. Cooke, F. Jahanian, D. Watson, and J. Nazario. The Blaster worm: Then and now. IEEE Security and Privacy, 03(4):26-31, 2005.

6. S. Blazy, Z. Dargaye, and X. Leroy. Formal verification of a C compiler front-end. In Proceedings of the 14th International Symposium on Formal Methods (FM'06), volume 4085 of Lecture Notes in Computer Science, pages 460-475, Hamilton, Canada, August 2006. Springer-Verlag.

7. B. Bray. Compiler security checks in depth, 2002. http://msdn2.microsoft.com/ en-us/library/aa290051 (vs.71) . aspx.

8. D. Brumley, T. Chiueh, R. Johnson, H. Lin, and D. Song. Efficient and accurate detection of integer-based attacks. In Proceedings of the 14th Annual Network and Distributed System Security Symposium (NDSS'07), San Diego, CA, USA, February 2007.

9. M. Castro, M. Costa, and T. Harris. Securing software by enforcing data-flow integrity. In Proceedings of the 7th conference on USENIX Symposium on Operating Systems Design and Implementation (OSDI'06), pages 11-11, Seattle, WA, USA, 2006. USENIX Association.

10. S. Chen, J. Xu, E.C. Sezer, P. Gauriar, and R. Iyer. Non-control-data attacks are realistic threats. In Proceedings of the Usenix Security Symposium, pages 177-192, Baltimore, MD, August 2005. USENIX Association.

11. Intel Corporation. Intel IA-32 architecture, software developer's manual, Volumes 1-3, 2007. http://developer.intel.com/design/Pentium4/documentation.htm.

12. C. Cowan, M. Barringer, S. Beattie, G. Kroah-Hartman, M. Frantzen, and J. Lokier. FormatGuard: Automatic protection from printf format string vulnerabilities. In Proceedings of the 10th USENIX Security Symposium, pages 191-200, Washington, DC, USA, August 2001. USENIX Association.

13. C. Cowan, C. Pu, D. Maier, H. Hinton, J. Walpole, P. Bakke, S. Beattie, A. Grier, P. Wagle, and Q. Zhang. StackGuard: Automatic adaptive detection and prevention of buffer-overflow attacks. In Proceedings of the 7th USENIX Security 
Symposium, pages 63-78, San Antonio, Texas, USA, January 1998. USENIX Association.

14. D. Dhurjati and V. Adve. Backwards-compatible array bounds checking for c with very low overhead. In Proceeding of the 28th international conference on Software engineering (ICSE '06), pages 162-171, Shanghai, China, 2006. ACM Press.

15. H. Etoh and K. Yoda. ProPolice -improved stack smashing attack detection. IPSJ SIGNotes Computer Security (CSEC), 14, October 2001.

16. E. Florio. GDIPLUS VULN - MS04-028 - CRASH TEST JPEG. full-disclosure at lists.netsys.com, 2004. Forum message, sent September 15.

17. J.C. Foster. Metasploit Toolkit for Penetration Testing, Exploit Development, and Vulnerability Research. Syngress Publishing, 2007.

18. M. Howard. Alleged bugs in Windows Vistas ASLR implementation, 2006. http://blogs.msdn.com/michael_howard/archive/2006/10/04/ Alleged-Bugs-in-Windows-Vista_1920_s-ASLR-Implementation . aspx.

19. M. Howard. Hardening stack-based buffer overrun detection in $\mathrm{VC}++$ 2005 SP1, 2007. http://blogs.msdn.com/michael_howard/archive/2007/04/03/ hardening-stack-based-buffer-overrun-detection-in-vc-2005-sp1.aspx.

20. M. Howard. Lessons learned from the animated cursor security bug, 2007. http://blogs.msdn.com/sdl/archive/2007/04/26/ lessons-learned-from-the-animated-cursor-security-bug.aspx.

21. M. Howard and S. Lipner. The Security Development Lifecycle. Microsoft Press, Redmond, WA, USA, 2006.

22. M. Howard and M. Thomlinson. Windows Vista ISV security, April 2007. http: //msdn2.microsoft. com/en-us/library/bb430720.aspx.

23. T. Jim, G. Morrisett, D. Grossman, M. Hicks, J. Cheney, and Y. Wang. Cyclone: A safe dialect of C. In USENIX Annual Technical Conference, pages 275-288, Monterey, California, U.S.A., June 2002. USENIX Association.

24. R. Jones and P. Kelly. Backwards-compatible bounds checking for arrays and pointers in C programs. In Proceedings of the 3rd International Workshop on Automatic Debugging, number 009-02 in Linköping Electronic Articles in Computer and Information Science, pages 13-26, Linköping, Sweden, 1997. Linköping University Electronic Press.

25. G. S. Kc, A.D. Keromytis, and V. Prevelakis. Countering code-injection attacks with instruction-set randomization. In Proceedings of the 10th ACM conference on Computer and communications security (CCS'03), pages 272-280, Washington D.C., USA, 2003. ACM Press.

26. V. Kiriansky, D. Bruening, and S. Amarasinghe. Secure execution via program shepherding. In Proceedings of the Usenix Security Symposium, pages 191-206, 2002.

27. Klog. The frame pointer overwrite. Phrack, 9(55), 1999.

28. X. Leroy. Formal certification of a compiler back-end, or: programming a compiler with a proof assistant. In 33rd symposium Principles of Programming Languages, pages 42-54. ACM Press, 2006.

29. D. Litchfield. Defeating the stack buffer overflow prevention mechanism of Microsoft Windows 2003 Server, 2003. http://www.nextgenss.com/papers/ defeating-w2k3-stack-protection.pdf.

30. B. Littlewood, P. Popov, and L. Strigini. Modeling software design diversity: a review. ACM Computing Surveys, 33(2):177-208, 2001.

31. G.C. Necula, S. McPeak, and W. Weimer. CCured: Type-safe retrofitting of legacy code. In Proceedings of the 29th ACM Symposium on Principles of Programming Languages (POPL'02), pages 128-139, Portland, OR, USA, January 2002. 
32. J. Newsome and D. Song. Dynamic taint analysis for automatic detection, analysis, and signature generation of exploits on commodity software. In Proceedings of the 12th Annual Network and Distributed System Security Symposium (NDSS'07), San Diego, CA, USA, February 2005.

33. PaX Project. The PaX project, 2004. http://pax.grsecurity.net/.

34. J. Pincus and B. Baker. Beyond stack smashing: Recent advances in exploiting buffer overruns. IEEE Security and Privacy, 2(4):20-27, 2004.

35. rix. Smashing C++ VPTRs. Phrack, 56, 2000.

36. H. Shacham. The geometry of innocent flesh on the bone: Return-into-libc without function calls (on the x86). In Proceedings of the 14th ACM conference on Computer and communications security, pages 552-561, Washington, D.C., U.S.A., October 2007. ACM, ACM Press.

37. H. Shacham, M. Page, B. Pfaff, E-J. Goh, N. Modadugu, and D. Boneh. On the effectiveness of address-space randomization. In Proceedings of the 11th ACM conference on Computer and communications security (CCS'04), pages 298-307, Washington DC, USA, 2004. ACM Press.

38. C. Small. A tool for constructing safe extensible $\mathrm{C}++$ systems. In Proceedings of the 3rd Conference on Object-Oriented Technologies and Systems, 1997.

39. E.H. Spafford. The Internet worm program: An analysis. SIGCOMM Computer Communication Review, 19(1):17-57, 1989.

40. Wikipedia. x86-64, 2007. http://en.wikipedia.org/wiki/X86-64.

41. Y. Younan. Efficient Countermeasures for Software Vulnerabilities due to Memory Management Errors. PhD thesis, Katholieke Universiteit Leuven, 2008.

42. Y. Younan, W. Joosen, and F. Piessens. Code injection in C and C++: A survey of vulnerabilities and countermeasures. Technical Report CW386, Departement Computerwetenschappen, Katholieke Universiteit Leuven, July 2004.

43. F. Zhou, J. Condit, Z. Anderson, I. Bagrak, R. Ennals, M. Harren, G.C. Necula, and E. Brewer. SafeDrive: Safe and recoverable extensions using language-based techniques. In Proceedings of the 7th conference on USENIX Symposium on Operating Systems Design and Implementation (OSDI'06), pages 4-4, Seattle, WA, USA, 2006. USENIX Association. 